\title{
ON EXISTENCE VARIETIES OF ORTHODOX SEMIGROUPS
}

\author{
J. DOYLE \\ (4 April 1991;24 March 1992) \\ Communicated by P. G. Trotter
}

\begin{abstract}
An existence variety of regular semigroups is a class of regular semigroups which is closed under the operations of forming all homomorphic images, all regular subsemigroups and all direct products. In this paper we generalize results on varieties of inverse semigroups to existence varieties of orthodox semigroups.
\end{abstract}

1991 Mathematics subject classification (Amer. Math. Soc.): 20M07, $20 \mathrm{M} 17$.

\section{Introduction}

An existence variety (or e-variety) of regular semigroups is a class of regular semigroups which is closed under the operations of forming of all homomorphic images, all regular subsemigroups and all direct products. This concept, which generalizes that of varieties of inverse semigroups, was introduced by T. E. Hall [7] and independently for orthodox semigroups by J. Kad'ourek and M. B. Szendrei [10] who called them bivarieties. In this paper we study e-varieties of orthodox semigroups.

In Section 2 we begin with preliminary results dealing mainly with congruences on regular or orthodox semigroups and with certain basic results on e-varieties of orthodox semigroups.

Let $\mathscr{G}$ denote the variety of groups, $\mathscr{O}$ the e-variety of orthodox semigroups and $\mathscr{L}_{e}(\mathscr{O})$ the lattice of e-varieties of orthodox semigroups. In Section 3 we show that the mapping $\phi: \mathscr{E} \mapsto \mathscr{E} \vee \mathscr{G}\left(\mathscr{E} \in \mathscr{L}_{e}(\mathscr{O})\right.$ is a complete lattice homomorphism and the $\nu_{1}=\phi \circ \phi^{-1}$-classes are complete modular sublattices of $\mathscr{L}_{\mathrm{e}}(\mathscr{O})$. Also the mapping $\psi: \mathscr{E} \mapsto \mathscr{E} \cap \mathscr{G}\left(\mathscr{E} \in \mathscr{L}_{e}(\mathscr{O})\right)$ is shown to be a lattice homomorphism and for each $v_{2}=\psi \circ \psi^{-1}$-class the join of all its members is $\mathscr{O}$.

In Section 4 several characterizations are given of completely semisimple (cryptic)

(C) 1995 Australian Mathematical Society 0263-6115/95 $\$ A 2.00+0.00$ 
e-varities of orthodox semigroups. Letting $v_{3}=v_{1} \cap v_{2}$, in Section 5 we show that the $v_{3}$-classes of cryptic e-varieties have maximum members and we identify the maximum member using a Mal'cev product.

In Section 6 we establish some properties of the Mal'cev product. In particular we show that the Mal'cev product respects the lattice operations in $\mathscr{L}_{e}(\mathscr{G})$, we prove an associativity result and we determine bi-identities for the Mal'cev product of a group variety and an e-variety of orthodox semigroups.

The results of this paper generalize analogous results on varieties of inverse semigroups by Kleiman [11], Reilly [19, 20] and Bales [1] (see also [18, Chapter XII]).

For undefined notation and terminology see [8].

\section{Preliminary Results}

Let $S$ be a regular semigroup. The set of idempotents is denoted by $E_{S}=E$ and for $a \in S$ denote by $V(a)$ the set of inverses of $a$ in $S$. We let $\sigma_{S}=\sigma$ denote the least group congruence, $\gamma_{S}=\gamma$ the least inverse semigroup congruence and $\mu_{S}=\mu$ the greatest idempotent separating (i.s.) congruence on $S$.

When $S$ is orthodox $\sigma, \gamma$ and $\mu$ are characterized by

$$
\begin{aligned}
\sigma= & \{(a, b) \in S \times S \mid e a e=e b e \text { for some } e \in E\} \\
\gamma= & \{(a, b) \in S \times S \mid V(a)=V(b)\} \\
\mu= & \left\{(a, b) \in S \times S \mid \text { there are inverses } a^{\prime} \in V(a), b^{\prime} \in V(b)\right. \text { such } \\
& \text { that } \left.a^{\prime} e a=b^{\prime} e b \text { and } a e a^{\prime}=b e b^{\prime} \text { for all } e \in E\right\}
\end{aligned}
$$

\section{([13, Theorems 4.4 and 5.1] and [14, Theorem 3.1]).}

Let $\mathscr{C}(S)$ denote the lattice of congruences on $S$. For any $\rho \in \mathscr{C}(S)$ the kernel and trace of $\rho$ are defined by

$$
\begin{aligned}
\operatorname{Ker} \rho & =\{a \in S \mid a \rho e \text { for some } e \in E\} \\
\operatorname{tr} \rho & =\rho \cap(E \times E) .
\end{aligned}
$$

For $\rho \in \mathscr{C}(S), \rho_{\min }$ and $\rho_{\max }$ denote the least, respectively greatest, congruence on $S$ such that $\operatorname{tr} \rho_{\min }=\operatorname{tr} \rho=\operatorname{tr} \rho_{\max }$.

THEOREM 2.1. [2, 4, Theorem 4.1] A congruence on a regular semigroup is uniquely determined by its kernel and trace.

LEMMA 2.2. [15, Lemma 2.5] Let $S$ be a regular semigroup and $\mathscr{F}$ be a nonempty family of congruences on $S$. Then 
(a) $\operatorname{Ker}\left(\bigcap_{\rho \in \mathscr{F}} \rho\right)=\bigcap_{\rho \in \mathscr{F}} \operatorname{Ker} \rho$

(b) $\operatorname{tr}\left(\bigcap_{\rho \in \mathscr{F}} \rho\right)=\bigcap_{\rho \in \mathscr{F}} \operatorname{tr} \rho$.

We call $S E$-unitary if $a \in S, e$ and $e a \in E$ implies that $a \in E$.

LEMMA 2.3. ([9, Lemma 2.6] or [23, Lemma 2.7]) Let $S$ be a regular semigroup. Then $S$ is $E$-unitary if and only if $E$ is a $\sigma$-class.

LEMMA 2.4. Let $S$ be a regular semigroup and $\rho, \xi \in \mathscr{C}(S)$. If $\rho \cap \sigma=\xi \cap \sigma$ then $\operatorname{tr} \rho=\operatorname{tr} \xi$. The converse holds if $S$ is E-unitary.

PROOF. The argument of [18, Theorem XII.2.1] applies to yield this result using Theorem 2.1 and Lemmas 2.2 and 2.3 at the appropriate places.

COROLLARY 2.5. Let $S$ be a regular semigroup and $\rho \in \mathscr{C}(S)$. If $S$ is $E$-unitary then $\rho_{\min }=\rho \cap \sigma$.

PROOF. As $\operatorname{tr} \rho=\operatorname{tr} \rho_{\min }, \rho \cap \sigma=\rho_{\min } \cap \sigma \subseteq \rho_{\min }$ by Lemma 2.4. Since we always have $\rho_{\min } \subseteq \rho \cap \sigma$ we have equality.

LEMMA 2.6. [16, page 196] Let $S$ be a regular semigroup and $\rho \in \mathscr{C}(S)$. Then $a \rho_{\max } b$ if and only if $(a \rho) \mu_{S / \rho}(b \rho)$.

LEMMA 2.7. Let $S$ and $T$ be regular semigroups and $\chi: S \rightarrow T$ be an i.s. homomorphism of $S$ onto $T$. Then for all $a, b \in S, a \mu_{S} b$ if and only if $(a \chi) \mu_{T}(b \chi)$.

ProOF. Let $\rho=\chi \circ \chi^{-1}$. Then $\rho$ is an i.s. congruence on $S$. Therefore $\rho \subseteq \mu_{S}$ and so $\rho_{\max }=\mu_{s}$. The result now follows easily from Lemma 2.6

THEOREM 2.8. [22, Theorem 3.4]; [24, Theorem 2.2] Let $S$ be a regular semigroup and $\theta$ be the convergence on $\mathscr{C}(S)$ induced by the mapping $\operatorname{tr}: \rho \mapsto \operatorname{tr} \rho \quad(\rho \in \mathscr{C}(S))$. Then for any $\rho \in \mathscr{C}(S), \rho \theta$ is a complete modular sublattice of $\mathscr{C}(S)$.

THEOREM 2.9. [12, Theorem 11] Let $S$ be an orthodox semigroup. Then the mapping defined by $\psi: \rho \mapsto \rho \vee \sigma \quad(\rho \in \mathscr{C}(S))$ is a homomorphism of $\mathscr{C}(S)$ onto the lattice of group congruences on $S$.

A regular subsemigroup $K$ of $S$ is called full if $E \subseteq K$ and self-conjugate if for all $a \in S, a^{\prime} \in V(a), a^{\prime} K a \subseteq K$. Let

$$
\begin{aligned}
& \mathscr{K}=\{K \subseteq S: \mid K \text { is a full self-conjugate regular subsemigroup of } S \text { and } \\
& \qquad K \subseteq \operatorname{Ker} \mu\}
\end{aligned}
$$


and for $K \in \mathscr{K}$ let

$$
\begin{array}{r}
\theta_{K}=\left\{(a, b) \in S \times S \mid \text { there are inverses } a^{\prime} \in V(a), b^{\prime} \in V(b)\right. \\
\text { such that } \left.a a^{\prime}=b b^{\prime}, a^{\prime} a=b^{\prime} b \text { and } a b^{\prime}, a^{\prime} b \in K\right\} .
\end{array}
$$

THEOREM 2.10. [3, Theorem 3.3] Let $S$ be an orthodox semigroup. Then the map $K \mapsto \theta_{K}$ is a one-to-one order preserving map of $\mathscr{K}$ onto the set of $i$. s. congruences on $S$ with inverse map $\theta \mapsto \operatorname{Ker} \theta$.

LEMMA 2.11. Let $S$ and $T$ be orthodox semigroups and $\chi: S \rightarrow T$ be a homomorphism of $S$ onto $T$. If $\theta$ is an i. s. congruence on $S$ then $\theta$ induces an i.s. congruence $\phi$ say on $T$ with kernel $(\operatorname{Ker} \theta) \chi$. Furthermore the following hold:

(a) for $a, b \in S$, if $a \theta$ then $(a \chi) \phi(b \chi)$;

(b) $T / \phi$ is a homomorphic image of $S / \theta$.

Note in particular that as $\gamma^{\natural}: S \rightarrow S / \gamma$ is a homomorphism of $S$ onto $S / \gamma$ an i.s. congruence $\theta$ on $S$ induces an i.s. congruence $\phi$ on $S / \gamma$ with kernel $(\operatorname{Ker} \theta) \gamma$.

PROOF. Let $K=\operatorname{Ker} \theta$. Then by Theorem $2.10, K$ is a full self-conjugate regular subsemigroup of $S$ and $K \subseteq \operatorname{Ker} \mu_{S}$. It is easily shown that $K \chi$ is a full regular subsemigroup of $T$ and $K \chi \subseteq \operatorname{Ker} \mu_{T}$. Furthermore $K \chi$ is self-conjugate, for if $x$ and $x^{\prime}$ are mutual inverses of $T$ then there are mutual inverses $a$ and $a^{\prime}$ of $S$ such that $x=a \chi$ and $x^{\prime}=a^{\prime} \chi$ by [5, Lemma 1] hence $x^{\prime}(K \chi) x=\left(a^{\prime} K a\right) \chi \subseteq K \chi$ as $K$ is self-conjugate. By Theorem $2.10, K \chi$ is the kernel of an i.s. congruence $\phi$ say on $T$.

Now (a) follows easily from the characterization of $\theta$ and $\phi$. To prove (b) define $\Phi: S / \theta \rightarrow T / \phi$ by $\Phi: a \theta \mapsto(a \chi) \phi$. Then $\Phi$ is well-defined by (a) and clearly $\Phi$ is a homomorphism of $S / \theta$ onto $T / \phi$.

THEOREM 2.12. Let $S$ be an orthodox semigroup. Then there exists a group $G, a$ subdirect product $T \subseteq S / \mu_{S} \times G$ and an i.s. homomorphism $\chi$ of $T$ onto $S$.

PROOF. Since every orthodox semigroup has an $E$-unitary cover [25, Theorem 5], the argument of [18, Theorem VII.4.8] applies to yield this result using Theorem 2.1 and Lemmas $2.3,2.7$ at the appropriate places.

We call $S$ completely semisimple if every principle factor of $S$ is completely 0 simple or completely simple.

LEMMA 2.13. Let $S$ be an orthodox semigroup. Then $S$ is completely semisimple if and only if $S / \gamma$ is completely semisimple. 
PROOF. Suppose $S / \gamma$ is completely semisimple and let $e, f \in E$ be such that $e \leq f$ and $e \mathscr{D} f$. Then $e \gamma \leq f \gamma$ and $e \gamma \mathscr{D} f \gamma$. As $S / \gamma$ is completely semisimple, [6, Result 6] yields that $e \gamma=f \gamma$. By the characterization of $\gamma, V(e)=V(f)$ and hence $f=f e f=e$ as $e \leq f$. By [6, Result 6], $S$ is completely semisimple.

Conversely, suppose $S$ is completely semisimple and let $e, f \in E$ be such that $e \gamma \leq f \gamma$ and $e \gamma \mathscr{D} f \gamma$. Then $e \gamma \mathscr{R} a \gamma \mathscr{L} f \gamma$ for some $a \in S$. Let $a^{\prime} \in V(a)$; then $e \gamma \mathscr{R} a a^{\prime} \gamma$ and it follows that $e \gamma=a a^{\prime} \gamma$. By the characterization of $\gamma, V(e)=V\left(a a^{\prime}\right)$ and it is easily shown that this implies that $e \mathscr{D} a a^{\prime}$; dually, $f \mathscr{D} a^{\prime} a$. Furthermore, as $e \gamma \leq f \gamma,(f e f) \gamma=e \gamma$ and so $f e f \mathscr{D} e$. Therefore fef $\mathscr{D} e \mathscr{D} a a^{\prime} \mathscr{D} a^{\prime} a \mathscr{D} f$ so that $f$ ef $\mathscr{D} f$; also it is clear that $f e f \leq f$. As $S$ is completely semisimple, [6, Result 6] yields that $f e f=f$. Now $e \gamma=(f e f) \gamma=f \gamma$ and by $[6$, Result 6$] S / \gamma$ is completely semisimple.

We call $S$ cryptic if the $\mathscr{H}$-relation is a congruence on $S$. Recall that a cryptic completely regular semigroup is a band of groups. Let $G=\cup\left\{H_{e} \mid e \in E\right\}$ be the union of the maximal subgroups of $S$.

LEMMA 2.14. Let $S$ be an orthodox semigroup. Then $S$ is cryptic if and only if $G$ is a band of groups.

Proof. Suppose $S$ is cryptic and let $a, b \in G$. Then $a \mathscr{H} e$ and $b \mathscr{H} f$ for some $e, f \in E$. Since $\mathscr{H}$ is a congruence on $S$ we have $a b \mathscr{H}$ ef and therefore $a b \in G$ as $e f \in E$ since $S$ is orthodox. Thus $G$ is a subsemigroup of $S$. As $G$ is a union of groups, $G$ is completely regular. By [17, Lemma IV.4.1] $\left.\mathscr{H}\right|_{G}$ is the $\mathscr{H}$-relation on $G$. Since $\mathscr{H}$ is a congruence on $S$ it follows that $\left.\mathscr{H}\right|_{G}$ is a congruence on $G$. Thus $G$ is a band of groups.

Conversely, suppose $G$ is a band of groups. Let $a, b \in S, a \mathscr{H} b$ and $c \in S$. Let $a^{\prime} \in V(a)$ and $b^{\prime} \in V(b)$; then $a a^{\prime} \mathscr{R} a \mathscr{R} b \mathscr{R} b b^{\prime}$ and so, as $a a^{\prime}$ and $b b^{\prime}$ are left identities of their $\mathscr{R}$-class we have

$$
a=b b^{\prime} a \text { and } \quad b=a a^{\prime} b .
$$

Also, as $a^{\prime} a \mathscr{L} a \mathscr{L} b \mathscr{L} b^{\prime} b$ and $a^{\prime} a, b^{\prime} b$ are right identities of their $\mathscr{L}$-class, we have

$$
a=a b^{\prime} b \quad \text { and } \quad b=b a^{\prime} a .
$$

By (1) and (2), $a^{\prime} b \cdot a^{\prime} a=a^{\prime} b$ and $a^{\prime} a b^{\prime} a \cdot a^{\prime} b=a^{\prime} a b^{\prime} b=a^{\prime} a$ so that $a^{\prime} a \mathscr{L} a^{\prime} b$. Also $a \mathscr{R} b$ implies $a^{\prime} a \mathscr{R} a^{\prime} b$; thus $a^{\prime} a \mathscr{H} a^{\prime} b$ and $a^{\prime} b \in G$. Let $c^{\prime} \in V(c)$; then as $\mathscr{H}$ is a congruence on $G$ we have that $a^{\prime} a c c^{\prime} \mathscr{H} a^{\prime} b c c^{\prime}$. Since $S$ is orthodox, $a^{\prime} a c c^{\prime} \in E$ and so $a^{\prime} a c c^{\prime}$ is the identity of $H_{a^{\prime} b c c^{\prime}}$ : therefore $a^{\prime} a c c^{\prime} a^{\prime} b c c^{\prime}=a^{\prime} b c c^{\prime}$. Now $a c \cdot c^{\prime} a^{\prime} b c=a a^{\prime} a c c^{\prime} a^{\prime} b c c^{\prime} c=a a^{\prime} b c c^{\prime} c=a a^{\prime} b c=b c$ by (1). By symmetry 
$b c \cdot c^{\prime} b^{\prime} a c=a c$ and so $a c \mathscr{R} b c$. Since $a \mathscr{L} b$ implies $a c \mathscr{L} b c$ we have that $a c \mathscr{H} b c$.

By (1) and (2), $a a^{\prime} \cdot b a^{\prime}=b a^{\prime}$ and $b a^{\prime} \cdot a b^{\prime} a a^{\prime}=b b^{\prime} a a^{\prime}=a a^{\prime}$ so that $a a^{\prime} \mathscr{R} b a^{\prime}$. Also $a \mathscr{L} b$ implies $a a^{\prime} \mathscr{L} b a^{\prime}$; thus $a a^{\prime} \mathscr{H} b a^{\prime}$ and $b a^{\prime} \in G$. As $\mathscr{H}$ is a congruence on $G, c^{\prime} c a a^{\prime} \mathscr{H} c^{\prime} c b a^{\prime}$ and as above we have $c^{\prime} c b a^{\prime} c^{\prime} c a a^{\prime}=c^{\prime} c b a^{\prime}$. Now $c b a^{\prime} c^{\prime} \cdot c a=$ $c c^{\prime} c b a^{\prime} c^{\prime} c a a^{\prime} a=c c^{\prime} c b a^{\prime} a=c b a^{\prime} a=c b$ by (2). By symmetry $c a b^{\prime} c^{\prime} \cdot c b=c a$ and so $c a \mathscr{L} c b$. Since $a \mathscr{R} b$ implies $c a \mathscr{R} c b$ we have that $c a \mathscr{H} c b$. Thus $\mathscr{H}$ is a congruence on $S$, that is, $S$ is cryptic.

We shall need some results and definitions from [10].

PROPOSITION 2.15. [10, Proposition 1.8] Let $\mathscr{C}$ be a nonempty class of orthodox semigroups. Then the e-variety generated by $\mathscr{C}$ consists of homomorphic images of regular subsemigroups of direct products of semigroups in $\mathscr{C}$.

The following is an easy consequence of the previous proposition.

COROLLARY 2.16. Let $\mathscr{E}$ and $\mathscr{F}$ be e-varieties of orthodox semigroups. Then the join $\mathscr{E} \vee \mathscr{F}$ of $\mathscr{E}$ and $\mathscr{F}$ consists of semigroups $S$ for which there exists $U \in \mathscr{E}$, $V \in \mathscr{F}$, a regular subdirect product $T \subseteq U \times V$ and a homomorphism $\varphi: T \rightarrow S$ of $T$ onto $S$.

Let $X$ be a nonempty set, ${ }^{*}$ be a bijection onto some set $X^{*}=\left\{x^{*} \mid x \in X\right\}$ disjoint from $X$ and let $F^{*}(X)$ be the free semigroup in the alphabet $X \cup X^{*}$ equipped with the involution * extending this bijection. Namely, for any word $u=y_{1} y_{2} \ldots y_{n}$ $\left(y_{i} \in X \cup X^{*}\right)$ we have $u^{*}=y_{n}^{*} \ldots y_{2}^{*} y_{1}^{*}$ with $\left(x^{*}\right)^{*}=x$ for $x \in X$.

A mapping $\chi: X \cup X^{*} \rightarrow S$ into an orthodox semigroup $S$ such that $x \chi, x^{*} \chi$ are mutual inverses in $S$ for all $x \in X$ will be called a matched mapping, as in [10].

By a bi-identity we mean any pair $u=v$ of words $u, v \in F^{*}(X)$. An orthodox semigroup $S$ satisfies the bi-identity $u=v$ if for any matched mapping $\chi: X \cup X^{*} \rightarrow$ $S$ we have $u \Phi=v \Phi$ where $\Phi: F^{*}(X) \rightarrow S$ is the unique homomorphism extending $\chi$. We say that a bi-identity is satisfied in a class $\mathscr{E}$ of orthodox semigroups if it is satsified by each member of $\mathscr{E}$. Let $u_{\alpha}=v_{\alpha}, \alpha \in A$ be a set of bi-identities. Then the class of all orthodox semigroups satisfying each bi-identity $u_{\alpha}=v_{\alpha}, \alpha \in A$ will be denoted by $\left[u_{\alpha}=v_{\alpha}\right]_{\alpha \in A}$.

We have the following Birkhoff type theorem for e-varieties of orthodox semigroups.

THEOREM 2.17. [10, Theorem 1.10] A class $\mathscr{E}$ of orthodox semigroups is an evariety if and only if there exists a set $u_{\alpha}=v_{\alpha}, \alpha \in A$ of bi-identities such that $\mathscr{E}=\left[u_{\alpha}=v_{\alpha}\right]_{\alpha \in A}$. 
For any $\mathscr{E} \in \mathscr{L}_{e}(\mathscr{O})$ let $\rho(\mathscr{E}, X)$ be the relation defined on $F^{*}(X)$ by $u \rho(\mathscr{E}, X) v$ if and only if $u=v$ is satisfied in $\mathscr{E}$. It is clear that $\rho(\mathscr{E}, X)$ is a congruence on $F^{*}(X)$. By the proof of $\left[10\right.$, Lemma 1.6] we have that $F \mathscr{O}(X)=F^{*}(X) / \rho(\mathscr{O}, X)$ is orthodox. Furthermore by Remark 2 following [10, Lemma 1.6], $F \mathscr{O}(X)$ is the free orthodox $*$-semigroup on the set $X$.

A congruence $\rho$ on a semigroup $S$ is called fully invariant if for every endomorphism $\alpha$ of $S$ and every $a, b \in S$ such that $a \rho b$ then $(a \alpha) \rho(b \alpha)$. Let $\mathscr{F} \mathscr{I}(S)$ denote the set of all fully invariant congruences on $S$.

LEMMA 2.18. Let $\mathscr{E} \in \mathscr{L}_{e}(\mathscr{O})$. Then the congruence $\rho(\mathscr{E}, X) / \rho(\mathscr{O}, X)$ on $F \mathscr{O}(X)$ is fully invariant.

PROOF. This follows from [10, Proposition 1.7] and the remark preceding [10, Proposition 1.7].

For each $\mathscr{E} \in \mathscr{L}_{e}(\mathscr{O})$ let $\rho(\mathscr{E})=\rho(\mathscr{E}, X) / \rho(\mathscr{O}, X)$ and for each $\rho \in \mathscr{F} \mathscr{I}(F \mathscr{O}(X))$ let $\mathscr{E}(\rho)=[u=v \mid(u \rho(\mathscr{O}, X)) \rho(v \rho(\mathscr{O}, X))]$.

THEOREM 2.19. Let $X$ be a countably infinite set and define two mappings $\rho$ and $\mathscr{E}$ by

$$
\begin{aligned}
& \rho: \mathscr{L}_{e}(\mathscr{O}) \rightarrow \mathscr{F} \mathscr{I}(F \mathscr{O}(X)): \mathscr{E} \mapsto \rho(\mathscr{E}) \\
& \mathscr{E}: \mathscr{F} \mathscr{I}(F \mathscr{O}(X)) \rightarrow \mathscr{L}_{e}(\mathscr{O}): \rho \mapsto \mathscr{E}(\rho) .
\end{aligned}
$$

Then $\rho$ and $\mathscr{E}$ are mutually inverse order anti-isomorphisms of $\mathscr{L}_{e}(\mathscr{O})$ and $\mathscr{F} \mathscr{I}(F \mathscr{O}(X))$.

PROOF. By [10, Corollary 1.12 and Remark] these two mappings determine a oneto-one correspondence between $\mathscr{L}_{e}(\mathscr{O})$ and $\mathscr{F} \mathscr{I}(F \mathscr{O}(X))$. It is not difficult to show that both maps invert the inclusion relation.

PROPOSITION 2.20. Let $\mathscr{E} \in \mathscr{L}_{e}(\mathscr{O})$ and $X$ be a countably infinite set. Then $\rho(\mathscr{E})$ is the least fully invariant congruence $\rho$ on $F \mathscr{O}(X)$ for which $F \mathscr{O}(X) / \rho \in \mathscr{E}$.

PROOF. Let $\rho \in \mathscr{F} \mathscr{I}(F \mathscr{O}(X))$. We show firstly that

(1) $u=v$ is satisfied by $F \mathscr{O}(X) / \rho$ if and only if $(u \rho(\mathscr{O}, X)) \rho(v \rho(\mathscr{O}, X))$.

Suppose $u=v$ is satisfied by $F \mathscr{O}(X) / \rho$. Define $\chi: X \cup X^{*} \rightarrow F \mathscr{O}(X) / \rho$ by $\chi: y \mapsto(y \rho(\mathscr{O}, X)) \rho \quad\left(y \in X \cup X^{*}\right)$; then $\chi$ is a matched mapping. Let $\Phi: F^{*}(X) \rightarrow F \mathscr{O}(X) / \rho$ be the unique homomorphism extending $\chi$. Since $u=v$ is satisfied by $F \mathscr{O}(X) / \rho$, we have that $u \Phi=v \Phi$. Clearly $u \Phi=(u \rho(\mathscr{O}, X)) \rho$ and $v \Phi=(v \rho(\mathscr{O}, X)) \rho$, and it follows that $(u \rho(\mathscr{O}, X)) \rho(v \rho(\mathscr{O}, X))$. 
Conversely, suppose $(u \rho(\mathscr{O}, X)) \rho(v \rho(\mathscr{O}, X))$. Let $\chi: X \cup X^{*} \rightarrow F \mathscr{O}(X) / \rho$ be any matched mapping and $\Phi: F^{*}(X) \rightarrow F \mathscr{O}(X) / \rho$ be the unique homomorphism extending $\chi$. To show that $u=v$ is satisfied by $F \mathscr{O}(X) / \rho$ we need to show that $u \Phi=$ $v \Phi$. Let $\bar{\Phi}: F \mathscr{O}(X) \rightarrow F \mathscr{O}(X) / \rho$ be the homomorphism induced by $\Phi$. Since $(x \rho(\mathscr{O}, X)) \bar{\Phi},\left(x^{*} \rho(\mathscr{O}, X)\right) \bar{\Phi}$ are mutual inverses in $F \mathscr{O}(X) / \rho$, by [5, Lemma 1] there are mutual inverses $\omega \rho(\mathscr{O}, X), \bar{\omega} \rho(\mathscr{O}, X)$ of $F \mathscr{O}(X)$ such that $(x \rho(\mathscr{O}, X)) \bar{\Phi}=$ $(\omega \rho(\mathscr{O}, X)) \rho$, and $\left(x^{*} \rho(\mathscr{O}, X)\right) \bar{\Phi}=(\bar{\omega} \rho(\mathscr{O}, X)) \rho($ where $\rho: F \mathscr{O}(X) \rightarrow F \mathscr{O}(X) / \rho$ is the natural homomorphism). Define $\psi: X \cup X^{*} \rightarrow F \mathscr{O}(X)$ by $\psi: x \mapsto$ $\omega \rho(\mathscr{O}, X), x^{*} \mapsto \bar{\omega} \rho(\mathscr{O}, X)$; then $\psi$ is a matched mapping. Let $\Psi: F^{*}(X) \rightarrow$ $F \mathscr{O}(X)$ be the unique homomorphism extending $\psi$ and $\bar{\Psi}: F \mathscr{O}(X) \rightarrow F \mathscr{O}(X)$ be the homomorphism induced by $\Psi$. It is easily seen that $\bar{\Phi}=\bar{\Psi} \rho$.

Since $\bar{\Psi}$ is an endomorphism of $F \mathscr{O}(X), \rho \in \mathscr{F} \mathscr{I}(F \mathscr{O}(X))$ and $(u \rho(\mathscr{O}, X)) \rho$ $(v \rho(\mathscr{O}, X))$ we obtain $((u \rho(\mathscr{O}, X)) \bar{\Psi}) \rho((v \rho(\mathscr{O}, X)) \bar{\Psi})$. Therefore

$$
\begin{aligned}
u \Phi & =(u \rho(\mathscr{O}, X)) \bar{\Phi}=(u \rho(\mathscr{O}, X)) \bar{\Psi} \rho=(v \rho(\mathscr{O}, X)) \bar{\Psi} \rho \\
& =(v \rho(\mathscr{O}, X)) \bar{\Phi}=v \Phi
\end{aligned}
$$

as required and (1) is proved.

By (1) $F \mathscr{O}(X) / \rho$ satisfies all bi-identities $u=v$ such that $(u \rho(\mathscr{O}, X)) \rho(v \rho(\mathscr{O}, X))$, so by the definition of $\mathscr{E}(\rho)$ we have

$$
F \mathscr{O}(X) / \rho \in \mathscr{E}(\rho)
$$

Now let $\mathscr{E} \in \mathscr{L}_{e}(\mathscr{O})$. Then $\rho(\mathscr{E}) \in \mathscr{F} \mathscr{I}(F \mathscr{O}(X))$ by Lemma 2.18. Therefore by (2), $F \mathscr{O}(X) / \rho(\mathscr{E}) \in \mathscr{E} \rho(\mathscr{E})$ and since $\mathscr{E} \rho$ is the identity map on $\mathscr{L}_{e}(\mathscr{O})$ (Theorem 2.19), we have that $F \mathscr{O}(X) / \rho(\mathscr{E}) \in \mathscr{E}$.

To show that $\rho(\mathscr{E})$ is minimal, let $\rho \in \mathscr{F} \mathscr{I}(F \mathscr{O}(X))$ be such that $F \mathscr{O}(X) / \rho \in \mathscr{E}$ and let $(u \rho(\mathscr{O}, X)) \rho(\mathscr{E})(v \rho(\mathscr{O}, X))$. Then $u \rho(\mathscr{E}, X) v$ so that $u=v$ is satisfied in $\mathscr{E}$. As $F \mathscr{O}(X) / \rho \in \mathscr{E}, u=v$ is satisfied by $F \mathscr{O}(X) / \rho$. Therefore, by (1), $(u \rho(\mathscr{O}, X)) \rho(v \rho(\mathscr{O}, X))$. Thus $\rho(\mathscr{E}) \subseteq \rho$ which proves the minimality of $\rho(\mathscr{E})$.

Proposition 2.21. Let $X$ be a countably infinite set and $\rho \in \mathscr{F} \mathscr{I}(F \mathscr{O}(X))$. Then $\rho_{\max } \in \mathscr{F} \mathscr{I}(F \mathscr{O}(X))$.

PROOF. The arguments of [16, Lemma 2.3] and [16, Theorem 4.2] with slight modifications apply to yield this result.

An orthodox semigroup is combinatorial if $\mathscr{H}$ is the identity congruence on $S$ or equivalently if all subgroups of $S$ are trivial.

PROPOSITION 2.22. The semigroup $F \mathscr{O}(X)$ is combinatorial and E-unitary. 
ProOF. Since $F \mathscr{O}(X)$ is the free orthodox $*$-semigroup, by [26, Theorem 2.2], $F \mathscr{O}(X)$ is combinatorial. A similar argument establishes that $F \mathscr{O}(X)$ is $E$-unitary.

\section{Joins and meets with groups}

In this section we show that the mappings $\phi: \mathscr{E} \mapsto \mathscr{E} \vee \mathscr{G}$ and $\psi: \mathscr{E} \mapsto \mathscr{E} \cap \mathscr{G}(\mathscr{E} \in$ $\mathscr{L}_{e}(\mathscr{O})$ ) are lattice homomorphisms, the former being complete, thus generalizing results of Kleiman [11]. These two homomorphisms induce two congruences $v_{1}$ and $v_{2}$ respectively on $\mathscr{L}_{e}(\mathscr{O})$. We show that the $v_{1}$ classes are complete modular sublattices of $\mathscr{L}_{e}(\mathscr{O})$ and that for each $\nu_{2}$ class the join of its members is $\mathscr{O}$.

PROPOSITION 3.1. For any $\mathscr{E}, \mathscr{F} \in \mathscr{L}_{e}(\mathscr{O})$ we have

$$
\mathscr{E} \vee \mathscr{G}=\mathscr{F} \vee \mathscr{G} \text { if and only if } \operatorname{tr} \rho(\mathscr{E})=\operatorname{tr} \rho(\mathscr{F}) \text {. }
$$

PROOF. Let $\sigma=\sigma_{F} \mathscr{O}_{(X)}$. Then using the characterization of $\sigma$ for orthodox semigroups it is easily seen that $\sigma$ is fully invariant. By Proposition $2.20, \rho(\mathscr{G})$ is the least fully invariant congruence $\rho$ on $F \mathscr{O}(X)$ for which $F \mathscr{O}(X) / \rho \in \mathscr{G}$ and hence it follows that $\sigma=\rho(\mathscr{G})$. Now using the anti-isomorphism $\rho$ of Theorem 2.19 we have

$$
\begin{aligned}
\mathscr{E} \vee \mathscr{G}=\mathscr{F} \vee \mathscr{G} & \text { if and only if } \rho(\mathscr{E}) \cap \rho(\mathscr{G})=\rho(\mathscr{F}) \cap \rho(\mathscr{G}) \\
& \text { if and only if } \rho(\mathscr{E}) \cap \sigma=\rho(\mathscr{F}) \cap \sigma \\
& \text { if and only if } \operatorname{tr} \rho(\mathscr{E})=\operatorname{tr} \rho(\mathscr{F})
\end{aligned}
$$

by Lemma 2.4 as $F \mathscr{O}(X)$ is $E$-unitary (Proposition 2.22 ).

A semigroup $S$ is called fundamental if $\mu$ is the identity congruence on $S$. Let $\mathscr{F} \mathscr{O}$ denote the class of fundamental orthodox semigroups.

THEOREM 3.2. For any $\mathscr{E}, \mathscr{F} \in \mathscr{L}_{e}(\mathscr{O})$ we have

$$
\mathscr{E} \vee \mathscr{G} \subseteq \mathscr{F} \vee \mathscr{G} \text { if and only if } \mathscr{E} \cap \mathscr{F} \mathscr{O} \subseteq \mathscr{F} \cap \mathscr{F} \mathscr{O}
$$

PROOF. The argument of [18, Theorem XII.2.4] applies to yield this result using Lemma 2.7, Theorem 2.12 and Corollary 2.16 at the appropriate places.

COROLlARY 3.3. For any $\mathscr{E}, \mathscr{F} \in \mathscr{L}_{e}(\mathscr{O})$ we have

$$
\mathscr{E} \vee \mathscr{G}=\mathscr{F} \vee \mathscr{G} \text { if and only if } \mathscr{E} \cap \mathscr{F} \mathscr{O}=\mathscr{F} \cap \mathscr{F} \mathscr{O}
$$


COROLlaRY 3.4. For any $\mathscr{E} \in \mathscr{L}_{e}(\mathscr{O})$ we have

$$
\mathscr{E} \vee \mathscr{G}=\{S \in \mathscr{O} \mid S / \mu \in \mathscr{E}\}
$$

PROOF. The argument of [18, Corollary XII.2.6] applies to yield this result using Theorem 2.12 and Corollary 3.3 at the appropriate places.

For any non-empty class $\mathscr{C}$ of orthodox semigroups, we let $\langle\mathscr{C}\rangle$ denote the e-variety generated by $\mathscr{C}$. For any $\mathscr{E}, \mathscr{F} \in \mathscr{L}_{e}(\mathscr{O})$ with $\mathscr{E} \subseteq \mathscr{F}$ we let $[\mathscr{E}, \mathscr{F}]$ denote the interval sublattice of $\mathscr{L}_{e}(\mathscr{O})$ with least element $\mathscr{E}$ and maximum element $\mathscr{F}$.

THEOREM 3.5. The mapping $\varphi: \mathscr{L}_{e}(\mathscr{O}) \rightarrow[\mathscr{G}, \mathscr{O}]$ defined by $\varphi: \mathscr{E} \mapsto \mathscr{E} \vee \mathscr{G}$ $\left(\mathscr{E} \in \mathscr{L}_{e}(\mathscr{O})\right)$ is a complete lattice homomorphism. Furthermore, let $v_{1}$ be the congruence on $\mathscr{L}_{e}(\mathscr{O})$ induced by $\varphi$. Then for each $\mathscr{E} \in \mathscr{L}_{e}(\mathscr{O}), \mathscr{E} v_{1}=[\langle\mathscr{E} \cap$ $\mathscr{F} \mathscr{O}\rangle, \mathscr{E} \vee \mathscr{G}]$ is a complete modular sublattice of $\mathscr{L}_{e}(\mathscr{O})$.

PROOF. From Corollary 3.4 it is easily shown that $\varphi$ is a complete lattice homomorphism. The theorem can be completed as in [18, Theorem XII.2.8] using Theorems 2.8, 2.19, Proposition 3.1 and Corollary 3.3 at the appropriate places.

PROPOSITION 3.6. Let $\mathscr{E} \in \mathscr{L}_{e}(\mathscr{O})$ and $\rho=\rho(\mathscr{E})$. Then $\mathscr{E}\left(\rho_{\min )}=\mathscr{E} \vee \mathscr{G}\right.$ and $\mathscr{E}\left(\rho_{\max }=\langle\mathscr{E} \cap \mathscr{F} \mathscr{O}\rangle\right.$. That is, $\rho_{\min }$ corresponds to the greatest and $\rho_{\max }$ to the least element of $\mathscr{E} \nu_{1}$.

Proof. By Lemma 2.18, $\rho \in \mathscr{F} \mathscr{I}(F \mathscr{O}(X))$. By Proposition 2.22, $F \mathscr{O}(X)$ is $E$ unitary and so $\rho_{\min }=\rho \cap \sigma$ by Corollary 2.5 (hence $\rho_{\min } \in \mathscr{F} \mathscr{I}(F \mathscr{O}(X))$ ). Therefore, using the anti-isomorphism $\mathscr{E}$ of Theorem $2.19, \mathscr{E}\left(\rho_{\text {min })}=\mathscr{E}(\rho \cap \sigma)=\mathscr{E}(\rho) \vee \mathscr{E}(\sigma)=\right.$ $\mathscr{E} \vee \mathscr{G}$.

By Proposition 2.21, $\rho_{\max } \in \mathscr{F} \mathscr{I}(F \mathscr{O}(X))$. Since $\langle\mathscr{E} \cap \mathscr{F} \mathscr{O}\rangle \vee \mathscr{G}=\mathscr{E} \vee \mathscr{G}$, $\operatorname{tr} \rho(\langle\mathscr{E} \cap \mathscr{F} \mathscr{O}\rangle)=\operatorname{tr} \rho(\mathscr{E})$ by Proposition 3.1. Therefore $\rho(\langle\mathscr{E} \cap \mathscr{F} \mathscr{O}\rangle) \subseteq \rho_{\max }$ and so $\mathscr{E}\left(\rho_{\max }\right) \subseteq \mathscr{E} \rho(\langle\mathscr{E} \cap \mathscr{F} \mathscr{O}\rangle)=\langle\mathscr{E} \cap \mathscr{F} \mathscr{O}\rangle$. Conversely, as $\operatorname{tr} \rho(\mathscr{E})=\operatorname{tr} \rho_{\max }$, $\mathscr{E} \vee \mathscr{G}=\mathscr{E}\left(\rho_{\text {max }}\right) \vee \mathscr{G}$ by Proposition 3.1, that is, $\mathscr{E}\left(\rho_{\text {max }}\right) \in \mathscr{E} v_{1}$. Therefore, as $\langle\mathscr{E} \cap \mathscr{F} \mathscr{O}\rangle$ is the minimum element of $\mathscr{E} v_{1},\langle\mathscr{E} \cap \mathscr{F} \mathscr{O}\rangle \subseteq \mathscr{E}\left(\rho_{\max }\right)$ we have equality.

LEMMA 3.7. The e-variety of orthodox semigroups is generated by its finite combinatorial members.

ProOF. By [26, Proposition 2.1], $F \mathscr{I}(X)=F \mathscr{O}(X) / \gamma$ is the free inverse semigroup on $X$. Consider the mapping $\chi: F \mathscr{O}(X) / \mathscr{J} \rightarrow F \mathscr{I}(X) / \mathscr{J}$ of the $\mathscr{J}$-classes of $F \mathscr{O}(X)$ onto the $\mathscr{J}$-classes of $F \mathscr{I}(X)$ defined by $\chi: J_{u \rho(\mathscr{O}, X)} \mapsto J_{u \rho(\mathscr{O}, X) \gamma}$. It is not difficult to show that $(u \rho(\mathscr{O}, X)) \mathscr{J}(v \rho(\mathscr{O}, X))$ if and only if $(u \rho(\mathscr{O}, X)) \gamma$ 
$\mathscr{J}(v \rho(\mathscr{O}, X)) \gamma$. It follows that $\chi$ is well-defined and one-to-one. Clearly $\chi$ and its inverse preserve the natural ordering of $\mathscr{J}$-classes.

For $u \rho(\mathscr{O}, X) \in F \mathscr{O}(X)$ let

$$
F_{u \rho(\mathscr{O}, X)}=\left\{v \rho(\mathscr{O}, X) \in F \mathscr{O}(X) \mid J_{u \rho(\mathscr{O}, X)} \subseteq J_{v \rho(\mathscr{O}, X)}\right\}
$$

and

$$
F_{u \rho(\mathscr{O}, X) \gamma}=\left\{v \rho(\mathscr{O}, X) \gamma \in F \mathscr{I}(X) \mid J_{u \rho(\mathscr{O}, X) \gamma} \subseteq J_{v \rho(\mathscr{O}, X) \gamma}\right\}
$$

By the first part of the proof of [18, Lemma XII.1.7] this latter set is finite. Since $\chi$ is an order preserving bijection of the $\mathscr{J}$-classes of $F \mathscr{O}(X)$ onto the $\mathscr{J}$-classes of $F \mathscr{I}(X)$ it follows that the number of $\mathscr{J}$-classes $J_{v \rho(\mathscr{O}, X)}$ such that $J_{u \rho(\mathscr{O}, X)} \subseteq J_{v \rho(\mathscr{O} . X)}$ is finite. By [26, Theorems 2.2, 4.4 and Proposition 4.7] each $\mathscr{J}$-class of $F \mathscr{O}(X)$ is finite and hence $F_{u \rho}(\mathscr{O}, X)$ is finite. Now the remaining part of the proof of [18, Lemma XII.1.7] yields the following result: if $\mathscr{E} \in \mathscr{L}_{e}(\mathscr{O})$ contains all finite combinatorial orthodox semigroups then $F \mathscr{O}(X) \in \mathscr{E}$. It follows that $\mathscr{E}$ contains all orthodox semigroups.

For any positive integer we let $\mathscr{C}_{n}=\left[x^{n}=x^{n+1}\right]$.

PROPOSITION 3.8. We have that $\bigvee_{n=1}^{\infty} \mathscr{C}_{n}=\mathscr{O}$.

PROOF. The argument of [18, Proposition XII.1.8] applies to yield this result using Lemma 3.7.

THEOREM 3.9. The mapping $\psi: \mathscr{L}_{e}(\mathscr{O}) \rightarrow \mathscr{L}(\mathscr{G})$ defined by $\psi: \mathscr{E} \mapsto \mathscr{E} \cap \mathscr{G}$ $\left(\mathscr{E} \in \mathscr{L}_{e}(\mathscr{O})\right)$ is a lattice homomorphism. Furthermore, let $v_{2}$ be the congruence on $\mathscr{L}_{e}(\mathscr{O})$ induced by $\psi$. Then for each $\mathscr{E} \in \mathscr{L}_{e}(\mathscr{O}), \mathscr{E} \cap \mathscr{G}$ is the least element of $\mathscr{E} v_{2}$, and $\mathscr{O}$ is the join of all members of $\mathscr{E} v_{2}$. Hence only $\mathscr{G} v_{2}$ has a greatest element.

PROOF. The argument of [18, Theorem XII.3.2] applies to yield this result using Theorems 2.9, 2.19 and Proposition 3.8 at the appropriate places.

\section{Completely semisimple and cryptic e-varieties}

An e-variety of orthodox semigroups is completely semisimple (cryptic) if all its members are completely semisimple (cryptic). In this section we characterize such e-varieties in several ways. We let $\mathscr{I}$ denote the variety of inverse semigroups.

PROPOSITION 4.1. Let $\mathscr{E}$ be a cryptic e-variety of orthodox semigroups. Then $\mathscr{E}$ is completely semisimple. 
PROOF. Since $\mathscr{E}$ is a cryptic e-variety of orthodox semigroups, $\mathscr{E} \cap \mathscr{I}$ is a cryptic variety of inverse semigroups. By [21, Corollary 5.13] $\mathscr{E} \cap \mathscr{I}$ is completely semisimple. Let $S \in \mathscr{E}$. Then $S / \gamma \in \mathscr{E} \cap \mathscr{I}$, and so $S / \gamma$ is completely semisimple. By Lemma $2.13, S$ is completely semisimple. Hence $\mathscr{E}$ is completely semisimple.

LEMMA 4.2. Let $S$ be an orthodox semigroup. Then $S \in \mathscr{C}_{n}$ if and only if $S / \gamma \in \mathscr{C}_{n}$.

Proof. Suppose $S / \gamma \in \mathscr{C}_{n}$ and let $a \in S$. Then, as $S / \gamma$ satisfies the identity $x^{n}=x^{n+1}$, we have that $a^{n} \gamma=(a \gamma)^{n}=(a \gamma)^{n+1}=a^{n+1} \gamma$, and so $V\left(a^{n}\right)=V\left(a^{n+1}\right)$. Let $a^{\prime} \in V(a)$. Then $\left(a^{\prime}\right)^{n} \in V\left(a^{n}\right)=V\left(a^{n+1}\right)$. Therefore $a^{n}=a^{n}\left(a^{\prime}\right)^{n} a^{n}=$ $a^{n}\left(a^{\prime}\right)^{n} a^{n+1}\left(a^{\prime}\right)^{n} a^{n}=a^{n}\left(a^{\prime}\right)^{n} a^{n} \cdot a\left(a^{\prime}\right)^{n} a^{n}=a^{n} a\left(a^{\prime}\right)^{n} a^{n}=a a^{n}\left(a^{\prime}\right)^{n} a^{n}=a a^{n}=a^{n+1}$. Thus $S$ satisfies the identity $x^{n}=x^{n+1}$ and so $S \in \mathscr{C}_{n}$. The converse implication is obvious.

An e-variety of orthodox semigroups is combinatorial if all its members are combinatorial.

We have the following characterization of combinatorial e-varieties of orthodox semigroups.

LEMMA 4.3. Let $\mathscr{E} \in \mathscr{L}_{e}(\mathscr{O})$. Then the following conditions are equivalent:

(a) $\mathscr{E}$ is combinatorial;

(b) $\mathscr{E} \cap \mathscr{I}$ is combinatorial;

(c) $\mathscr{E} \subseteq \mathscr{C}_{n}$ for some positive integer $n$;

(d) $\mathscr{E} \cap \mathscr{G}=\mathscr{T}$ - the variety of all trivial groups.

PROOF. Clearly (a) implies (b).

(b) implies (c). Suppose $\mathscr{E} \cap \mathscr{I}$ is combinatorial. By [18, Lemma XII.1.10] $\mathscr{E} \cap \mathscr{I} \subseteq \mathscr{C}_{n}$ for some positive integer $n$. Now it follows easily from Lemma 4.2 that $\mathscr{E} \subseteq \mathscr{C}_{n}$.

(c) implies (d). Suppose $\mathscr{E} \subseteq \mathscr{C}_{n}$. Then $\mathscr{E} \cap \mathscr{G} \subseteq \mathscr{C}_{n} \cap \mathscr{G}=\mathscr{T}$ since any group which satisfies the identiy $x^{n}=x^{n+1}$ must be trivial.

(d) implies (a). Suppose $\mathscr{E} \cap \mathscr{G}=\mathscr{T}$ and let $S \in \mathscr{E}$. Then all subgroups of $S$ are trivial, that is, $S$ is combinatorial. Hence $\mathscr{E}$ is combinatorial.

LEMMA 4.4. Let $\mathscr{E} \in \mathscr{L}_{e}(\mathscr{O})$. Then

$$
\mathscr{E} \subseteq\left[x^{n} x^{* n}=x^{2 n} x^{* 2 n}\right] \text { if and only if } \mathscr{E} \cap \mathscr{I} \subseteq\left[x^{n} x^{-n}=x^{-n} x^{n}\right]
$$

PROOF. Suppose $\mathscr{E} \subseteq\left[x^{n} x^{* n}=x^{2 n} x^{* 2 n}\right]$; let $S \in \mathscr{E} \cap \mathscr{I}$, and $a \in S$. Then $a^{n} a^{-n}=$ $a^{n}\left(a^{-n} a^{n}\right) a^{-n}=a^{n}\left(a^{-2 n} a^{2 n}\right) a^{n}=\left(a^{n} a^{-n}\right)\left(a^{-n} a^{n}\right)\left(a^{n} a^{-n}\right)=\left(a^{-n} a^{n}\right)\left(a^{n} a^{-n}\right)\left(a^{n} a^{-n}\right)=$ 
$\left(a^{-n} a^{n}\right)\left(a^{n} a^{-n}\right)=\left(a^{-n} a^{n}\right)\left(a^{-n} a^{n}\right)\left(a^{n} a^{-n}\right)=\left(a^{-n} a^{n}\right)\left(a^{n} a^{-n}\right)\left(a^{-n} a^{n}\right)=a^{-n}\left(a^{2 n} a^{-2 n}\right)$. $a^{n}=a^{-n} a^{n} a^{-n} a^{n}=a^{-n} a^{n}$. That is, $S$ satisfies the inverse semigroup identity $x^{n} x^{-n}=$ $x^{-n} x^{n}$ and hence $\mathscr{E} \cap \mathscr{I} \subseteq\left[x^{n} x^{-n}=x^{-n} x^{n}\right]$.

Conversely, suppose $\mathscr{E} \cap \mathscr{I} \subseteq\left[x^{n} x^{-n}=x^{-n} x^{n}\right]$ and let $S \in \mathscr{E}, a \in S$ and $a^{\prime} \in V(a)$. Since $S / \gamma \in \mathscr{E} \cap \mathscr{I}, S / \gamma$ satisfies the inverse semigroup identity $x^{n} x^{-n}=$ $x^{-n} x^{n}$. Therefore $\left(a^{n}\left(a^{\prime}\right)^{n}\right) \gamma=(a \gamma)^{n}\left(a^{\prime} \gamma\right)^{n}=(a \gamma)^{n}(a \gamma)^{-n}=(a \gamma)^{-n}(a \gamma)^{n}=$ $\left(\left(a^{\prime}\right)^{n} a^{n}\right) \gamma$ and so $V\left(a^{n}\left(a^{\prime}\right)^{n}\right)=V\left(\left(a^{\prime}\right)^{n} a^{n}\right)$. Then, as $a^{n}\left(a^{\prime}\right)^{n} \in V\left(a^{n}\left(a^{\prime}\right)^{n}\right)=$ $V\left(\left(a^{\prime}\right)^{n} a^{n}\right)$,

$$
\begin{aligned}
a^{n}\left(a^{\prime}\right)^{n} & =a^{n}\left(a^{\prime}\right)^{n} a^{n}\left(a^{\prime}\right)^{n}=a^{n}\left(a^{\prime}\right)^{n} a^{n} a^{n} \cdot\left(a^{\prime}\right)^{n}\left(a^{\prime}\right)^{n} a^{n}\left(a^{\prime}\right)^{n} \\
& =a^{n} a^{n}\left(a^{\prime}\right)^{n}\left(a^{\prime}\right)^{n}=a^{2 n}\left(a^{\prime}\right)^{2 n}
\end{aligned}
$$

That is, $S$ satisfies the bi-identity $x^{n} x^{* n}=x^{2 n} x^{* 2 n}$ and hence $\mathscr{E} \subseteq\left[x^{n} x^{* n}=x^{2 n} x^{* 2 n}\right]$.

For any $\mathscr{E} \in \mathscr{L}_{e}(\mathscr{O})$ we let $T(\mathscr{E})=\{S \in \mathscr{E} \mid\langle S\rangle$ is combinatorial $\}$. It is easily seen that $T(\mathscr{E}) \cap \mathscr{I}=T(\mathscr{E} \cap \mathscr{I})$

We denote the bicyclic semigroup by $\mathscr{C}$.

We can now give characterizations of completely semisimple e-varieties of orthodox semigroups.

THEOREM 4.5. Let $\mathscr{E} \in \mathscr{L}_{e}(\mathscr{O})$. Then the following conditions are equivalent:

(a) $\mathscr{C} \notin \mathscr{E}$;

(b) $\mathscr{E}$ is completely semisimple;

(c) $\mathscr{E} \cap \mathscr{I}$ is completely semisimple;

(d) $\mathscr{E} \subseteq\left[x^{n} x^{* n}=x^{2 n} x^{* 2 n}\right]$ for some positive integer $n$;

(e) $T(\mathscr{E}) \subseteq \mathscr{C}_{n}$ for some positive integer $n$;

(f) $T(\mathscr{E})$ is an e-variety.

Moreover, the least positive integer satisfying (d) coincides with the least positive integer satisfying (e).

PROOF. The equivalence of (a) and (b) follows easily from [20, Theorem 3.4] and Lemma 2.13. Clearly (b) implies (c).

(c) implies (d). Suppose $\mathscr{E} \cap \mathscr{I}$ is completely semisimple. By [20, Theorem 3.4], $\mathscr{E} \cap \mathscr{I} \subseteq\left[x^{n} x^{-n}=x^{-n} x^{n}\right]$ for some positive integer $n$. Therefore $\mathscr{E} \subseteq\left[x^{n} x^{* n}=\right.$ $x^{2 n} x^{* 2 n}$ ] by Lemma 4.4

(d) implies (e). Suppose $\mathscr{E} \subseteq\left[x^{n} x^{* n}=x^{2 n} x^{* 2 n}\right]$ and let $S \in T(\mathscr{E})$. Then $S \in \mathscr{E}$ and $\langle S\rangle$ is combinatorial. Clearly then $S / \gamma \in \mathscr{E} \cap \mathscr{I}$ and $\langle S / \gamma\rangle$ is combinatorial so that $S / \gamma \in T(\mathscr{E} \cap \mathscr{I})$. By Lemma 4.4, $\mathscr{E} \cap \mathscr{I} \subseteq\left[x^{n} x^{-n}=x^{-n} x^{n}\right]$ and hence $T(\mathscr{E} \cap \mathscr{I}) \subseteq \mathscr{C}_{n}$ by [20, Theorem 3.4]. Therefore $S / \gamma \in \mathscr{C}_{n}$ and also $S \in \mathscr{C}_{n}$ by Lemma 4.2. Thus $T(\mathscr{E}) \subseteq \mathscr{E}_{n}$. 
(e) implies (f). Suppose $T(\mathscr{E}) \subseteq \mathscr{C}_{n}$ and let $S \in \mathscr{E} \cap \mathscr{C}_{n}$. Then $\langle S\rangle \in \mathscr{C}_{n}$ and so $\langle S\rangle$ is combinatorial by Lemma 4.3. Therefore $S \in T(\mathscr{E})$ whence $\mathscr{E} \cap \mathscr{C}_{n} \subseteq T(\mathscr{E})$. The opposite inclusion is obvious: thus $T(\mathscr{E})=\mathscr{E} \cap \mathscr{C}_{n}$ is an e-variety.

(f) implies (a). Suppose $T(\mathscr{E})$ is an e-variety. Then $T(\mathscr{E} \cap \mathscr{I})=T(\mathscr{E}) \cap \mathscr{I}$ is a variety of inverse semigroups. By [20, Theorem 3.4], $\mathscr{C} \notin \mathscr{E} \cap \mathscr{I}$ and hence $\mathscr{C} \notin \mathscr{E}$.

Now let $n$ be the least positive integer such that $\mathscr{E} \subseteq\left[x^{n} x^{* n}=x^{2 n} x^{* 2 n}\right]$ and $m$ be the least positive integer such that $T(\mathscr{E}) \subseteq \mathscr{C}_{m}$. Since $\mathscr{E} \subseteq\left[x^{n} x^{* n}=x^{2 n} x^{* 2 n}\right]$ if and only if $\mathscr{E} \cap \mathscr{I} \subseteq\left[x^{n} x^{-n}=x^{-n} x^{n}\right], n$ is the least positive integer such that $\mathscr{E} \cap \mathscr{I} \subseteq\left[x^{n} x^{-n}=x^{-n} x^{n}\right]$ and since $T(\mathscr{E}) \subseteq \mathscr{C}_{m}$ if and only if $T(\mathscr{E} \cap \mathscr{I}) \subseteq \mathscr{C}_{m}, m$ is the least positive integer such that $T(\mathscr{E} \cap \mathscr{I}) \subseteq \mathscr{C}_{m}$. By [20, Theorem 3.4], $m=n$.

Before characterizing cryptic e-varieties of orthodox semigroups we prove

LEMMA 4.6. We have $\mathscr{C}_{n} \vee \mathscr{G}=\left[x^{n} y y^{*} x^{* n}=x^{n+1} y y^{*} x^{* n+1}\right]$.

PROOF. let $S \in\left[x^{n} y y^{*} x^{* n}=x^{n+1} y y^{*} x^{* n+1}\right], a \in S, a^{\prime} \in V(a)$ and $e \in E$. Then $a^{n} e\left(a^{\prime}\right)^{n}=a^{n+1} e\left(a^{\prime}\right)^{n+1}$ and $\left(a^{\prime}\right)^{n} e a^{n}=\left(a^{\prime}\right)^{n+1} e a^{n+1}$. By the characterization of $\mu$ this gives $a^{n} \mu a^{n+1}$ and it follows that $S / \mu \in \mathscr{C}_{n}$. By Corollary 3.4, $S \in \mathscr{C}_{n} \vee \mathscr{G}$ and so $\left[x^{n} y y^{*} x^{* n}=x^{n+1} y y^{*} x^{* n+1}\right] \subseteq \mathscr{C}_{n} \vee \mathscr{G}$. The opposite inclusion is straightforward and we have equality.

THEOREM 4.7. Let $\mathscr{E} \in \mathscr{L}_{e}(\mathscr{O})$. The following conditions are equivalent:

(a) $\mathscr{E}$ is cryptic;

(b) $\mathscr{E} \cap \mathscr{I}$ and $\mathscr{E} \cap \mathscr{C} \mathscr{R}$ are cryptic, where $\mathscr{C} \mathscr{R}$ is the variety of completely regular semigroups;

(c) $\mathscr{E} \subseteq \mathscr{C}_{n} \vee \mathscr{G}$ for some positive integer $n$;

(d) $\mathscr{E} \subseteq\left[x^{n} y y^{*} x^{* n}=x^{n+1} y y^{*} x^{* n+1}\right]$ for some positive integer $n$;

(e) $\mathscr{E} \cap \mathscr{F} \mathscr{O} \subseteq \mathscr{C}_{n}$ for some positive integer $n$;

(f) $T(\mathscr{E})=\mathscr{E} \cap \mathscr{F} \mathscr{O}$;

(g) $\mathscr{E} \cap \mathscr{F} \mathscr{O}$ is a combinatorial e-variety.

PROOF. Clearly (a) implies (b).

(b) implies (a). Suppose $\mathscr{E} \cap \mathscr{I}$ and $\mathscr{E} \cap \mathscr{C} \mathscr{R}$ are cryptic and let $S \in \mathscr{E}$. We show first that if $a \gamma \mathscr{H} e \gamma$, where $a \in S$ and $e \in \mathscr{E}$, then $a \in G$, the union of the maximal subgroups of $S$. Let $a^{\prime} \in V(a)$ then $a^{\prime} \gamma=(a \gamma)^{-1}$ and so $a a^{\prime} \gamma=a^{\prime} a \gamma=e \gamma$. Therefore, by the characterization of $\gamma, V\left(a a^{\prime}\right)=V\left(a^{\prime} a\right)=V(e)$. Also, as $a e \gamma=$ $e a \gamma=a \gamma, V(a e)=V(e a)=V(a)$. Since

$$
\begin{aligned}
a a^{\prime} e a^{\prime} a \cdot e a & =a a^{\prime} e a & & \left(\text { since } e \in V(e)=V\left(a^{\prime} a\right)\right) \\
& =a & & \left(\text { since } a^{\prime} e \in V(e a)=V(a)\right)
\end{aligned}
$$


and

$$
\begin{aligned}
a e \cdot a a^{\prime} e a^{\prime} a & =a e a^{\prime} a & & \left(\text { since } e \in V(e)=V\left(a a^{\prime}\right)\right) \\
& =a & & \text { (since } \left.e a^{\prime} \in V(a e)=V(a)\right)
\end{aligned}
$$

it follows that $a \mathscr{H} a a^{\prime} e a^{\prime} a$ and so $a \in G$.

Let $a, b \in G$ then $a \mathscr{H} e$ and $b \mathscr{H} f$ for some $e, f \in E$. Then $a \gamma \mathscr{H} e \gamma$ and by $\mathscr{H} f \gamma$. Since $S / \gamma \in \mathscr{E} \cap \mathscr{I}$ which is cryptic, $\mathscr{H}$ is a congruence on $S / \gamma$ and so $(a b) \gamma \mathscr{H}(e f) \gamma$. By the previous paragraph $a b \in G$ and so $G$ is a subsemigroup of $S$. As $G$ is a union of groups, $G$ is a completely regular subsemigroup of $S$, therefore $G \in \mathscr{E} \cap \mathscr{C} \mathscr{R}$. Since $\mathscr{E} \cap \mathscr{C} \mathscr{R}$ is cryptic we have that $G$ is a band of groups. By Lemma $2.14, S$ is cryptic and hence $\mathscr{E}$ is cryptic.

(a) implies (c). Suppose $\mathscr{E}$ is a cryptic e-variety. Then, by Lemma $4.1, \mathscr{E}$ is also completely semisimple. By Theorem $4.5 \mathscr{E}^{\circ} \subseteq\left[x^{n} x^{* n}=x^{2 n} x^{* 2 n}\right]$ for some positive integer $n$. Let $S \in \mathscr{E}, a \in S$ and $a^{\prime} \in V(a)$. Then $a^{n}=a^{n}\left(a^{\prime}\right)^{n} a^{n}=a^{2 n}\left(a^{\prime}\right)^{2 n} a^{n}=$ $a^{n+1} \cdot a^{n-1}\left(a^{*}\right)^{2 n} a^{n}$ and $a^{n+1}=a^{n} \cdot a$ which implies that $a^{n} \mathscr{R} a^{n+1}$. Analagously $a^{n} \mathscr{L} a^{n+1}$ and so $a^{n} \mathscr{H} a^{n+1}$. Since $S$ is cryptic, $\mathscr{H}$ is a congruence on $S$ so that $\mathscr{H}=\mu$. Therefore $a^{n} \mu a^{n+1}$ and it follows that $S / \mu \in \mathscr{C}_{n}$. By Corollary 3.4 $S \in \mathscr{C}_{n} \vee \mathscr{G}$ and hence $\mathscr{E} \subseteq \mathscr{C}_{n} \vee \mathscr{G}$

The equivalence of (c) and (d) follows from Lemma 4.6.

(c) implies (e). Suppose $\mathscr{E} \subseteq \mathscr{C}_{n} \vee \mathscr{G}$. Then $\mathscr{E} \cap \mathscr{F} \mathscr{O} \subseteq\left(\mathscr{C}_{n} \vee \mathscr{G}\right) \cap \mathscr{F} \mathscr{O}=\mathscr{C}_{n} \cap \mathscr{F} \mathscr{O}$ by Corollary 3.3 as $\left(\mathscr{C}_{n} \vee \mathscr{G}\right) \vee \mathscr{G}=\mathscr{C}_{n} \vee \mathscr{G}$. Since $\mathscr{C}_{n}$ is combinatorial and in particular fundamental, $\mathscr{C}_{n} \cap \mathscr{F} \mathscr{O}=\mathscr{C}_{n}$, whence $\mathscr{E} \cap \mathscr{F} \mathscr{O} \subseteq \mathscr{C}_{n}$.

The arguments of [20, Theorem 3.9] applies to show that (e) implies (f) and (f) implies ( $\mathrm{g})$.

(g) implies (a). Suppose $\mathscr{E} \cap \mathscr{F} \mathscr{O}$ is a combinatorial e-variety and let $S \in \mathscr{E}$. Then $S / \mu \in \mathscr{E} \cap \mathscr{F} \mathscr{O}$. As $\mathscr{E} \cap \mathscr{F} \mathscr{O}$ is a combinatorial e-variety, $S / \mu$ has no nontrivial subgroups. Therefore $\mu=\mathscr{H}$ is a congruence. That is, $S$ is cryptic, and hence $\mathscr{E}$ is cryptic.

REMARK 1 . Note that the conditions $\mathscr{E}$ is cryptic and $\mathscr{E} \cap \mathscr{I}$ is cryptic are not equivalent. For let $\mathscr{O} \mathscr{G}$ be the variety of orthodox completely regular semigroups. Then $\mathscr{O} \mathscr{G}$ is not cryptic. However, $\mathscr{O G} \cap \mathscr{I}=\mathscr{S} \mathscr{G}$; the variety of semilattices of groups is cryptic.

Let $\mathscr{C} \mathscr{S}$ and $\mathscr{C} \mathscr{S} \mathscr{H}$ denote respectively the classes of completely semisimple and cryptic orthodox semigroups. Neither of these classes is an e-variety. Denote by $\mathscr{L}_{e}(\mathscr{C S})$ and $\mathscr{L}_{e}(\mathscr{C} \mathscr{S} \mathscr{H})$ the set of all e-varieties contained in $\mathscr{C} \mathscr{S}$ and $\mathscr{C} \mathscr{S} \mathscr{H}$ respectively.

COROLlARY 4.8. Both $\mathscr{L}_{e}(\mathscr{C} \mathscr{S})$ and $\mathscr{L}_{e}(\mathscr{C} \mathscr{S} \mathscr{H})$ are ideals of $\mathscr{L}_{e}(\mathscr{O})$. 
PROOF. Let $\mathscr{E}, \mathscr{F} \in \mathscr{L}_{e}(\mathscr{C} \mathscr{S})$. By Theorem $4.5, \mathscr{E} \subseteq\left[x^{n} x^{* n}=x^{2 n} x^{* 2 n}\right]$ and $\mathscr{F} \subseteq\left[x^{m} x^{* m}=x^{2 m} x^{* 2 m}\right]$ for some positive integers $m$ and $n$. Since $\mathscr{E}$ satisfies $x^{n} x^{* n}=x^{2 n} x^{* 2 n}$, it is easily seen that $\mathscr{E}$ satisfies $x^{n} x^{* n}=x^{k n} x^{* k n}$ for any positive integer $k$, and it follows that $\mathscr{E}$ satisfies $x^{m n} x^{* m n}=x^{2 m n} x^{* 2 m n}\left(=x^{m} x^{* m}\right)$. It follows that $\mathscr{E} \vee \mathscr{F} \subseteq\left[x^{m n} x^{* m n}=x^{2 m n} x^{* 2 m n}\right]$ and hence by Theorem 4.5, that $\mathscr{E} \vee \mathscr{F} \in$ $\mathscr{L}_{e}(\mathscr{C} \mathscr{S})$.

If $\mathscr{E} \in \mathscr{L}_{e}(\mathscr{C S})$ and $\mathscr{F} \in \mathscr{L}_{e}(\mathscr{O})$ then it follows easily from Theorem 4.5 that $\mathscr{E} \cap \mathscr{F} \in \mathscr{L}_{e}(\mathscr{C} \mathscr{S})$. Consequently $\mathscr{L}_{e}(\mathscr{C} \mathscr{S})$ is an ideal of $\mathscr{L}_{e}(\mathscr{O})$. The second assertion of the corollary is easily proved from Theorem 4.7

For any $\mathscr{U} \in \mathscr{L}(\mathscr{G})$ we let $Q(\mathscr{U})=\left\{\mathscr{E} \in \mathscr{L}_{e}(\mathscr{O}) \mid \mathscr{E} \cap \mathscr{G}=\mathscr{U}\right\}$. It is easily shown, from Theorem 3.9, that $Q(\mathscr{U})$ is a sublattice of $\mathscr{L}_{e}(\mathscr{O})$ for each $\mathscr{U} \in \mathscr{L}(\mathscr{G})$.

CoROLlaRY 4.9. Let $\mathscr{U} \in \mathscr{L}(\mathscr{G})$ and $\mathscr{E} \in Q(\mathscr{T})$. Then $\mathscr{E} \vee \mathscr{U} \in \mathscr{C} \mathscr{S} \mathscr{H}$.

PROOF. Since $\mathscr{E}$ is combinatorial, $\mathscr{E} \subseteq \mathscr{C}_{n}$ for some positive integer $n$ by Lemma 4.3. Therefore $\mathscr{E} \vee \mathscr{U} \subseteq \mathscr{C}_{n} \vee \mathscr{G}$ and hence $\mathscr{E} \vee \mathscr{U} \in \mathscr{C} \mathscr{S} \mathscr{H}$ by Theorem 4.7.

LEMMA 4.10. Let $\mathscr{U} \in \mathscr{L}(\mathscr{G})$. Then the mapping $\chi: Q(\mathscr{T}) \rightarrow Q(\mathscr{U}): \mathscr{E} \mapsto$ $\mathscr{E} \vee \mathscr{U}$ is an isomorphism of $Q(\mathscr{T})$ onto $Q(\mathscr{U})$.

ProOF. Let $\mathscr{E} \in Q(\mathscr{T})$. By Theorem 3.9, $(\mathscr{E} \vee \mathscr{U}) \cap \mathscr{G}=(\mathscr{E} \cap \mathscr{G}) \vee(\mathscr{U} \cap \mathscr{G})=\mathscr{U}$ so that $\chi$ maps $Q(\mathscr{T})$ into $Q(\mathscr{U})$. Define $\Phi: Q(\mathscr{T}) \chi \rightarrow Q(\mathscr{T})$ by $\Phi: \mathscr{E} \mapsto$ $\mathscr{E} \cap \mathscr{F} \mathscr{O}(\mathscr{E} \in Q(\mathscr{T}) \chi)$. Note that as $\mathscr{E} \in Q(\mathscr{T}) \chi, \mathscr{E} \in \mathscr{C} \mathscr{S} \mathscr{K}$ by Corollary 4.9 and hence $\mathscr{E} \cap \mathscr{F} \mathscr{O}$ is a combinatorial e-variety by Theorem 4.7. It is easily shown, from Corollary 3.3, that $\chi \Phi$ and $\Phi \chi$ are identity maps on their domains. Since both $\chi, \Phi$ are obviously order preserving it follows that both $\chi, \Phi$ are lattice isomorphisms.

COROLlaRY 4.11. The mapping $\chi: \mathscr{E} \mapsto \mathscr{E} \vee \mathscr{G}(\mathscr{E} \in Q(\mathscr{T}))$ is an isomorphism of $Q(\mathscr{T})$ onto $Q(\mathscr{G}) \cap \mathscr{L}_{e}(\mathscr{C} \mathscr{S} \mathscr{H})$ with inverse $\chi^{-1}: \mathscr{E} \mapsto \mathscr{E} \cap \mathscr{F} \mathscr{O}$.

PrOOF. The argument of [20, Corollary 3.14] applies to yield this result using Corollary 3.4, Theorems 4.5, 4.7 and Lemma 4.10 at the appropriate places.

COROLlaRY 4.12. The mapping $\Phi: \mathscr{L}_{e}(\mathscr{C} \mathscr{S} \mathscr{H}) \rightarrow Q(\mathscr{T}): \mathscr{E} \mapsto \mathscr{E} \cap \mathscr{F} \mathscr{O}$ is a homomorphism of $\mathscr{L}_{e}(\mathscr{C} \mathscr{H} \mathscr{S})$ onto $Q(\mathscr{T})$.

PROOF. The argument of [20, Corollary 3.15] applies to yield this result using Corollary 3.4 and Theorems 3.5, 4.5 and 4.11 at the appropriate places. 


\section{The maximum element in a $v_{3}$-class}

Recall that the mappings $\phi: \mathscr{E} \mapsto \mathscr{E} \vee \mathscr{G}$ and $\psi: \mathscr{E} \mapsto \mathscr{E} \cap \mathscr{G}\left(\mathscr{E} \in \mathscr{L}_{e}(\mathscr{O})\right)$ induce congruences $v_{1}$ and $v_{2}$ respectively on $\mathscr{L}_{e}(\mathscr{O})$. Thus for $\mathscr{E}, \mathscr{F} \in \mathscr{L}_{e}(\mathscr{O})$

$$
\begin{aligned}
& \mathscr{E} \nu_{1} \mathscr{F} \text { if and only if } \mathscr{E} \vee \mathscr{G}=\mathscr{F} \vee \mathscr{G} ; \text { and } \\
& \mathscr{E} \nu_{2} \mathscr{F} \text { if and only if } \mathscr{E} \cap \mathscr{G}=\mathscr{F} \cap \mathscr{G} .
\end{aligned}
$$

By Corollary 3.3 we have an alternate description of $v_{1}$ namely

$$
\mathscr{E} v_{1} \mathscr{F} \text { if and only if } \mathscr{E} \cap \mathscr{F} \mathscr{O}=\mathscr{F} \cap \mathscr{F} \mathscr{O}
$$

Let $v_{3}=v_{1} \cap v_{2}$. Then it is not difficult to show that each $v_{3}$-class has a minimum member. In this section we show that each $v_{3}$-class of a cryptic e-variety also has a maximum member. (This result is not true in general: see for example $[19$, Section 5].)

PROPOSITION 5.1. Let $V$ be a $v_{3}$-class and $\mathscr{E} \in V$. Then $(\mathscr{E} \cap \mathscr{G}) \vee\langle\mathscr{E} \cap \mathscr{F} \mathscr{O}\rangle$ is the minimum member of $V$.

PROOF. The argument of [19, Proposition 3.1] applies to yield this result.

To identify the maximum member of a $v_{3}$-class of a cryptic e-variety we will need the concept of a Mal'cev product for e-varieties.

Let $\mathscr{E}$ and $\mathscr{F}$ be any classes of orthodox semigroups. The class of all orthodox semigroups $S$ for which there exists a congruence $\theta$ on $S$ such that $S / \theta \in \mathscr{F}$ and $e \theta \in \mathscr{E}$ for each $e \in \mathscr{E}$ is called the Mal' cev product of $\mathscr{E}$ and $\mathscr{F}$ denoted $\mathscr{E} \circ \mathscr{F}$.

Note that if $\mathscr{E}$ and $\mathscr{F}$ are classes of inverse semigroups, then $\mathscr{E} \circ \mathscr{F}$ is a class of inverse semigroups. For let $S \in \mathscr{E} \circ \mathscr{F}, \theta$ be a congruence as in the definition of the Mal'cev product and $e, f \in E$. Then, as $S / \theta$ is an inverse semigroup, $(e f) \theta=(f e) \theta$ and therefore, as each idempotent $\theta$-class is an inverse semigroup, effe $=f e e f$, that is, $e f e=f e f$. Hence $e f=e f e f=f e f f=f f e f=f e f e=f e$ and so $S$ is an inverse semigroup.

The Mal'cev product of e-varieties is not in general an e-variety (see for example [1, Example 1.2]). However it does have the following property.

LEMMA 5.2. Let $\mathscr{E}, \mathscr{F} \in \mathscr{L}_{e}(\mathscr{O})$. The $\mathscr{E} \circ \mathscr{F}$ is closed under direct products and regular subsemigroups.

PrOOF. The argument of [18, Lemma XII.8.2] applies to yield this result. 
PROPOSITION 5.3. Let $\mathscr{U} \in \mathscr{L}(\mathscr{G})$ and $\mathscr{E} \in \mathscr{L}_{e}(\mathscr{O})$. Then $\mathscr{U} \circ \mathscr{E}$ is an e-variety of orthodox semigroups.

PROOF. By Lemma 5.2, it remains to show that $\mathscr{U} \circ \mathscr{E}$ is closed under homomorphic images. Let $S \in \mathscr{U} \circ \mathscr{E}$ and $\chi: S \rightarrow T$ be a homomorphism of $S$ onto an orthodox semigroup $T$. Let $\theta$ be a congruence on $S$ such that $S / \theta \in \mathscr{E}$ and $e \theta \in \mathscr{U}$ for each $e \in E$. Since $e \theta \in \mathscr{U}$ a group variety, $e \theta$ contains a unique idempotent for each $e \in E$. Thus $\theta$ is an i.s. congruence. By Lemma 2.11, $\theta$ induces an i.s. congruence, $\phi$ say, on $T$. Furthermore, $T / \phi$ is a homomorphic image of $S / \theta$. Thus $T / \phi \in \mathscr{E}$ as $S / \theta \in \mathscr{E}$

As $K=\operatorname{Ker} \theta=\bigcup_{e \in E} e \theta$ is a band of groups in $\mathscr{U}, K / \gamma_{K}$ is a semilattice of groups in $\mathscr{U}$. By the proof of [8, Theorem IV.2.1], $K / \gamma_{K}$ is a strong semilattice of groups in $\mathscr{U}$ and hence $K / \gamma_{K} \in \mathscr{U} \vee \mathscr{S}$ by [8, Theorem IV.5.12], where $\mathscr{S}$ is the variety of semilattices. It is easily shown that the mapping $\Phi: K \rightarrow K / \gamma_{K} \times E$, defined by $\Phi: a \mapsto\left(a \gamma_{K}, e\right)$ where $a \in K$ and $a \theta e$, is an embedding. It follows that $K \in \mathscr{U} \vee \mathscr{B}$ and hence $K \chi \in \mathscr{U} \vee \mathscr{B}$, where $\mathscr{B}$ is the variety of bands. Since $K \chi=\operatorname{Ker} \theta$ and $\phi$ is i.s., all idempotent $\phi$-classes are in $(\mathscr{U} \vee \mathscr{B}) \cap \mathscr{G}$. By Theorem $3.9(\mathscr{U} \vee \mathscr{B}) \cap \mathscr{G}=(\mathscr{U} \cap \mathscr{G}) \vee(\mathscr{B} \cap \mathscr{G})=\mathscr{U}$. Thus $e \phi \in \mathscr{U}$ for each $e \in E_{T}$. We have that $\phi$ is a congruence on $T$ such that $T / \phi \in \mathscr{E}$ and $e \phi \in \mathscr{U}$ for each $e \in E_{T}$. Thus $T \in \mathscr{U} \circ \mathscr{E}$, whence $\mathscr{U} \circ \mathscr{E}$ is an e-variety.

THEOREM 5.4. Let $\mathscr{E} \in \mathscr{L}_{e}(\mathscr{C} \mathscr{S} \mathscr{H})$. Then $(\mathscr{E} \cap \mathscr{G}) \circ(\mathscr{E} \cap \mathscr{F} \mathscr{O})$ is the maximum member of $\mathscr{E} v_{3}$.

PROOF. Let $\mathscr{U}=\mathscr{E} \cap \mathscr{G}$ and $\mathscr{F}=\mathscr{E} \cap \mathscr{F} \mathscr{O}$ (which is an e-variety by Theorem 4.7). The first part of the proof of [19, Theorem 3.5] applies to show that $\mathscr{U} \circ \mathscr{F}$ contains $\mathscr{E} \nu_{3}$.

We need to show that $\mathscr{U} \circ \mathscr{F} \in \mathscr{E} \nu_{3}$.

Let $G \in(\mathscr{U} \circ \mathscr{F}) \cap \mathscr{G}$. Then there is a congruence $\theta$ on $G$ such that $G / \theta \in \mathscr{F}$ and $1 \theta \in \mathscr{U}$. Since $G / \theta$ is a group in $\mathscr{F} \subseteq \mathscr{F} \mathscr{O}, \theta$ must be the universal congruence. Then $G=1 \theta=\mathscr{U}$, and so $(\mathscr{U} \circ \mathscr{F}) \cap \mathscr{G} \subseteq \mathscr{U}=\mathscr{E} \cap \mathscr{G}$. The opposite inclusion is easily proved and we have equality. Hence $\mathscr{U} \circ \mathscr{F} \in \mathscr{E} v_{2}$.

The theorem may now be completed as in [19, Theorem 3.5].

COROLlaRY 5.5. Let $\mathscr{E} \in \mathscr{L}_{e}(\mathscr{C} \mathscr{S} \mathscr{H})$. Then $\mathscr{E} v_{3}=[(\mathscr{E} \cap \mathscr{G}) \vee(\mathscr{E} \cap \mathscr{F} \mathscr{O}),(\mathscr{E} \cap$ $\mathscr{G}) \circ(\mathscr{E} \cap \mathscr{F} \mathscr{O})]$ is a complete modular lattice.

PROOF. This follows easily from Proposition 5.1, Theorem 5.4 and Theorem 3.5, as $v_{3}$-classes are sublattices of $v_{1}$-classes. 
COROLlary 5.6. Let $\mathscr{U} \in \mathscr{L}(\mathscr{G})$ and $\mathscr{E} \in Q(\mathscr{T})$ then $[\mathscr{U} \vee \mathscr{E}, \mathscr{U} \circ \mathscr{E}]$ is a $v_{3}$-class.

PROOF. Let $\mathscr{F}=\mathscr{U} \vee \mathscr{E}$. Then $\mathscr{F} \in \mathscr{L}_{e}(\mathscr{C} \mathscr{S} \mathscr{H})$ by Corollary 4.9. By Corollary 5.5 the $\nu_{3}$-class containing $\mathscr{F}$ is $[(\mathscr{F} \cap \mathscr{G}) \vee(\mathscr{F} \cap \mathscr{F} \mathscr{O}),(\mathscr{F} \cap \mathscr{G}) \circ(\mathscr{F} \cap \mathscr{F} \mathscr{O})]$. Since $\mathscr{F} \vee \mathscr{G}=\mathscr{E} \vee \mathscr{G}$, Corollary 3.3 yields that $\mathscr{F} \cap \mathscr{F} \mathscr{O}=\mathscr{E} \cap \mathscr{F} \mathscr{O}=\mathscr{E}$, while Theorem 3.9 yields that $\mathscr{F} \cap \mathscr{G}=\mathscr{U}$. Therefore the $v_{3}$-class containing $\mathscr{F}$ is $[\mathscr{U} \vee \mathscr{E}, \mathscr{U} \circ \mathscr{E}$ ].

\section{Further results on the Mal'cev product}

In this section we show that the Mal'cev product respects the lattice operations in $\mathscr{L}(\mathscr{G})$, we establish an associativity result and we determine bi-identities for the Mal'cev product of a group variety and an e-variety of orthodox semigroups.

LEMMA 6.1. Let $\mathscr{U} \in \mathscr{L}(\mathscr{G})$ and $\mathscr{E} \in \mathscr{L}_{e}(\mathscr{O})$. Then

(a) $\mathscr{U} \circ \mathscr{E}=((\mathscr{U} \circ \mathscr{E}) \cap \mathscr{I}) \vee \mathscr{E}$;

(b) $(\mathscr{U} \circ \mathscr{E}) \cap \mathscr{I}=\mathscr{U} \circ(\mathscr{E} \cap \mathscr{I})$.

PROOF. (a) Let $S \in \mathscr{U} \circ \mathscr{E}$. Then $S / \gamma \in(\mathscr{U} \circ \mathscr{E}) \cap \mathscr{I}$. Let $\theta$ be a congruence on $S$ such that $S / \theta \in \mathscr{E}$ and $e \theta \in \mathscr{U}$ for each $e \in E$. Then $\theta$ is an i.s. congruence on $S$. Therefore $\theta \subseteq \mu$ and so $S / \mu \cong(S / \theta) /(\mu / \theta) \in \mathscr{E}$. By [8, p. 191], $\gamma \cap \mu=\imath$ : therefore $S$ is a subdirect product of $S / \gamma$ and $S / \mu$. Hence $S \in((\mathscr{U} \circ \mathscr{E}) \cap \mathscr{I}) \vee \mathscr{E}$, and so $\mathscr{U} \circ \mathscr{E} \subseteq((\mathscr{U} \circ \mathscr{E}) \cap \mathscr{I}) \cap \mathscr{E}$. The converse inclusion is obvious.

(b) Since the Mal'cev product of classes of inverse semigroups is a class of inverse semigroups it is clear that $\mathscr{U} \circ(\mathscr{E} \cap \mathscr{I}) \subseteq(\mathscr{U} \circ \mathscr{E}) \cap \mathscr{I}$. The converse inclusion is trivial.

LEMMA 6.2. Let $\left\{\mathscr{U}_{\alpha} \mid \alpha \in A\right\}$ be a family of varieties of groups and $\mathscr{E} \in \mathscr{L}_{e}(\mathscr{O})$. Then

$$
\left(\bigvee \mathscr{U}_{\alpha}\right) \circ \mathscr{E}=\bigvee\left(\mathscr{U}_{\alpha} \circ \mathscr{E}\right) \text { and }\left(\bigcap \mathscr{U}_{\alpha}\right) \circ \mathscr{E}=\bigcap\left(\mathscr{U}_{\alpha} \circ \mathscr{E}\right)
$$

PROOF. By Lemma 6.1 and [19, Lemma 4.1] we have

$$
\begin{aligned}
\left(\bigvee \mathscr{U}_{\alpha}\right) \circ \mathscr{E} & =\left(\left(\left(\bigvee \mathscr{U}_{\alpha}\right) \circ \mathscr{E}\right) \cap \mathscr{I}\right) \vee \mathscr{E} \\
& =\left(\left(\bigvee \mathscr{U}_{\alpha}\right) \circ(\mathscr{E} \cap \mathscr{I})\right) \vee \mathscr{E} \\
& =\left(\bigvee\left(\mathscr{U}_{\alpha} \circ(\mathscr{E} \cap \mathscr{I})\right)\right) \vee \mathscr{E} \\
& =(\bigvee((\mathscr{U} \circ \mathscr{E}) \cap \mathscr{I})) \vee \mathscr{E}
\end{aligned}
$$




$$
\begin{aligned}
& =\bigvee\left(\left(\left(\mathscr{U}_{\alpha} \circ \mathscr{E}\right) \cap \mathscr{I}\right) \vee \mathscr{E}\right) \\
& =\bigvee\left(\mathscr{U}_{\alpha} \circ \mathscr{E}\right) .
\end{aligned}
$$

The argument of the corresponding assertion of [19, Lemma 4.1] applies to establish that $\left(\bigcap \mathscr{U}_{\alpha}\right) \circ \mathscr{E}=\bigcap\left(\mathscr{U}_{\alpha} \circ \mathscr{E}\right)$.

THEOREM 6.3. If $\mathscr{E} \in \mathscr{L}_{e}(\mathscr{O})$ is such that $\mathscr{G} \subseteq \mathscr{E}$ then the mapping $\mathscr{U} \mapsto \mathscr{U} \circ \mathscr{E}$ is a complete lattice isomorphism of $\mathscr{L}(\mathscr{G})$ into $[\mathscr{E}, \mathscr{G} \vee \mathscr{E}]$.

PROOF. The argument of [19, Theorem 4.3] applies to yield this result.

THEOREM 6.4. Let $\mathscr{U}, \mathscr{V} \in \mathscr{L}(\mathscr{G})$ and $\mathscr{E} \in \mathscr{L}_{e}(\mathscr{O})$. Then $(\mathscr{U} \circ \mathscr{V}) \circ \mathscr{E}=$ $\mathscr{U} \circ(\mathcal{V} \circ \mathscr{E})$

PROOF. By Lemma 6.1 and [1, Theorem 4.4] we have

$$
\begin{aligned}
(\mathscr{U} \circ \mathscr{V}) \circ \mathscr{E} & =(((\mathscr{U} \circ \mathscr{V}) \circ \mathscr{E}) \cap \mathscr{I}) \vee \mathscr{E} \\
& =((\mathscr{U} \circ \mathscr{V}) \circ(\mathscr{E} \cap \mathscr{I})) \vee \mathscr{E} \\
& =(\mathscr{U} \circ(\mathscr{V} \circ(\mathscr{E} \cap \mathscr{I}))) \vee \mathscr{E} \\
& =(\mathscr{U} \circ((\mathscr{V} \circ \mathscr{E}) \cap \mathscr{I})) \vee \mathscr{E} \\
& =((\mathscr{U} \circ(\mathscr{V} \circ \mathscr{E})) \cap \mathscr{I}) \vee \mathscr{E} \\
& =((\mathscr{U} \circ(\mathscr{V} \circ \mathscr{E})) \cap \mathscr{I}) \vee(\mathscr{V} \circ \mathscr{E}) \\
& =\mathscr{U} \circ(\mathscr{V} \circ \mathscr{E})
\end{aligned}
$$

where the second last equality follows since $\mathscr{V} \subseteq \mathscr{U} \circ \mathscr{V}$ and hence $\mathscr{V} \circ \mathscr{E} \subseteq$ $(\mathscr{U} \circ \mathcal{V}) \circ \mathscr{E}$.

We now determine bi-identities for the Mal'cev product $\mathscr{G} \circ \mathscr{E}$ where $\mathscr{E} \in \mathscr{L}_{e}(\mathscr{O})$.

Let $X$ be a countably infinite set. We will use the notation $\bar{x}$ to denote a string of elements $x_{1}, x_{1}^{*}, \ldots, x_{n}, x_{n}^{*}$ of $X \cup X^{*}$. Note that any bi-identity $u(\bar{x})=v(\bar{x})$ is equivalent to the the three bi-identities $u(\bar{x}) v^{*}(\bar{x})=v(\bar{x}) v^{*}(\bar{x}), v^{*}(\bar{x}) u(\bar{x})=$ $v^{*}(\bar{x}) v(\bar{x})$ and $u(\bar{x}) v^{*}(\bar{x}) u(\bar{x}) u^{*}(\bar{x}) u^{*}(\bar{x})=u(\bar{x}) u^{*}(\bar{x})$. For suppose $S$ is an orthodox semigroup which satisfies the latter set of bi-identities and let $\bar{s}=s_{1}, s_{1}^{\prime}, \ldots, s_{n}$, $s_{n}^{\prime}$ be a string of elements of $S$ where $s_{i}^{\prime} \in V\left(s_{i}\right)$. Then $u(\bar{s})=u(\bar{s}) u^{*}(\bar{s}) u(\bar{s})=$ $u(\bar{s}) v^{*}(\bar{s}) u(\bar{s}) u^{*}(\bar{s}) u(\bar{s})=u(\bar{s}) v^{*}(\bar{s}) u(\bar{s})=u(\bar{s}) v^{*}(\bar{s}) v(\bar{s})=v(\bar{s}) v^{*}(\bar{s}) v(\bar{s})=v(\bar{s})$, that is, $S$ satisfies the bi-identity $u(\bar{x})=v(\bar{x})$. The converse is clear. Thus for any $\mathscr{E} \in \mathscr{L}_{e}(\mathscr{O})$ there is a basis of bi-identities of the form $v(\bar{x})=i(\bar{x})$ where $i(x) \rho(\mathscr{O}, X)$ is an idempotent of $F \mathscr{O}(X)$. If we write

$$
\begin{aligned}
\operatorname{Idem}(\mathscr{E})= & \left\{(v(\bar{x}), i(\bar{x})) \in F^{*}(X) \times F^{*}(X) \mid \mathscr{E}\right. \text { satisfies the bi-identity } \\
& v(\bar{x})=i(\bar{x}) \text { and } i(\bar{x}) \rho(\mathscr{O}, X) \text { is an idempotent of } F \mathscr{O}(X)\}
\end{aligned}
$$


then $\operatorname{Idem}(\mathscr{E})$ provides a basis of bi-identities for $\mathscr{E}$.

THEOREM 6.5. Let $\mathscr{E} \in \mathscr{L}_{e}(\mathscr{O})$. Then the following are equivalent:

(a) $S \in \mathscr{G} \circ \mathscr{E}$;

(b) $S / \mu \in \mathscr{E}$;

(c) $S$ satisfies all bi-identities of the following forms

$$
v(\bar{x}) v^{*}(\bar{x}) i(\bar{x})=i(\bar{x})=i(\bar{x}) v^{*}(\bar{x}) v(\bar{x}),
$$

where $(v(\bar{x}), i(\bar{x})) \in \operatorname{Idem}(\mathscr{E})$.

PROOF. The equivalence of (a) and (b) is not difficult to prove and is left to the reader.

(b) implies (c). Suppose $S / \mu \in \mathscr{E}$ and let $(v(\bar{x}), i(\bar{x})) \in \operatorname{Idem}(\mathscr{E})$ and $\bar{s}=s_{1}$, $s_{1}^{\prime}, \ldots, s_{n}, s_{n}^{\prime}$ be a string of elements of $S$ where $s_{i}^{\prime} \in V\left(s_{i}\right)$. As $s_{i} \mu \in S / \mu$, $s_{i}^{\prime} \mu \in V\left(s_{i} \mu\right), S / \mu \in \mathscr{E}$ and $\mathscr{E}$ satisfies the bi-identity $v(\bar{x})=i(\bar{x})$, we have that $v\left(s_{1} \mu, s_{1}^{\prime} \mu, \ldots, s_{n} \mu, s_{n}^{\prime} \mu\right)=i\left(s_{1} \mu, s_{1}^{\prime} \mu, \ldots, s_{n} \mu, s_{n}^{\prime} \mu\right)$, so that $v(\bar{s}) \mu=i(\bar{s}) \mu$ or $v(\bar{s}) \mu i(\bar{s})$. Since $\mu \subseteq \mathscr{H}$, in particular $v(\bar{s})$ and $i(\bar{s})$ are $\mathscr{H}$-related elements of $S$. As $i(\bar{s})$ is an idempotent of $S$, the $\mathscr{H}$-class $H_{i(\bar{s})}$ is a group. Let $v(\bar{s})^{-1}$ be the inverse of $v(\bar{s})$ in $H_{i(\bar{s})}$. Then $v(\bar{s}) v^{*}(\bar{s}) i(\bar{s})=v(\bar{s}) v^{*}(\bar{s}) v(\bar{s}) v(\bar{s})^{-1}=v(\bar{s}) v(\bar{s})^{-1}=i(\bar{s})$, and similarly $i(\bar{s})=i(\bar{s}) v^{*}(\bar{s}) v(\bar{s})$. Thus $S$ satisfies all bi-identities of the forms given in (c).

(c) implies (b). Suppose $S$ satisfies all bi-identities of the forms given in (c). Since Idem $(\mathscr{E})$ provides a basis of bi-identities for $\mathscr{E}$, we need to show that $S / \mu$ satisfies $v(\bar{x})=i(\bar{x})$, where $(v(\bar{x}), i(\bar{x})) \in \operatorname{Idem}(\mathscr{E})$. Now, if $a \in S / \mu$ and $\alpha^{\prime} \in$ $V(a)$, then by [5, Lemma 1] there are $s \in S$ and $s^{\prime} \in V(s)$ such that $a=s \mu$ and $a^{\prime}=s^{\prime} \mu$. Therefore we need to show that $v(\bar{s} \mu)=i(\bar{s} \mu)$, or equivalently, that $v(\bar{s}) \mu i(\bar{s})$, where $s=s_{1}, s_{1}^{\prime}, \ldots, s_{n}, s_{n}^{\prime}$ is a string of elements of $S$ with $s_{i}^{\prime} \in V\left(s_{i}\right)$. We first prove the following result: if $(v(\bar{x}), i(\bar{x})) \in \operatorname{Idem}(\mathscr{E})$, then $v(\bar{s}) \mathscr{H} i(\bar{s})$. Since $(v(\bar{x}), i(\bar{x})) \in \operatorname{Idem}(\mathscr{E})$, by assumption, we have that $v(\bar{s}) v^{*}(\bar{s}) i(\bar{s})=i(\bar{s})=$ $i(\bar{s}) v^{*}(\bar{s}) v(\bar{s})$. Also, it is clear that $\left(v^{*}(\bar{x}) i(\bar{x}), v^{*}(\bar{x}) v(\bar{x})\right) \in \operatorname{Idem}(\mathscr{E})$ and again, by assumption, we have that

$$
v^{*}(\bar{s}) i(\bar{s}) i^{*}(\bar{s}) v(\bar{s}) v^{*}(\bar{s}) v(\bar{s})=v^{*}(\bar{s}) v(\bar{s})=v^{*}(\bar{s}) v(\bar{s}) i^{*}(\bar{s}) v(\bar{s}) v^{*}(\bar{s}) i(\bar{s}) .
$$

Therefore $i(\bar{s}) i^{*}(\bar{s}) v(\bar{s})=v(\bar{s}) v^{*}(\bar{s}) i(\bar{s}) i^{*}(\bar{s}) v(\bar{s}) v^{*}(\bar{s}) v(\bar{s})=v(\bar{s}) v^{*}(\bar{s}) v(\bar{s})=v(\bar{s})$ and $v(\bar{s}) i^{*}(\bar{s}) i(\bar{s})=v(\bar{s}) v^{*}(\bar{s}) v(\bar{s}) i^{*}(\bar{s}) v(\bar{s}) v^{*}(\bar{s}) i(\bar{s})=v(\bar{s}) v^{*}(\bar{s}) v(\bar{s})=v(\bar{s})$. Now $v(\bar{s}) v^{*}(\bar{s}) i(\bar{s})=i(\bar{s})$ and $i(\bar{s}) i^{*}(\bar{s}) v(\bar{s})=v(\bar{s})$ show that $v(\bar{s}) \mathscr{R} i(\bar{s})$, and $i(\bar{s}) v^{*}(\bar{s})$ $v(\bar{s})=i(\bar{s})$ and $v(\bar{s}) i^{*}(\bar{s}) i(\bar{s})=v(\bar{s})$ show that $v(\bar{s}) \mathscr{L} i(\bar{s})$. Thus $v(\bar{s}) \mathscr{H} i(\bar{s})$.

To show that $v(\bar{s}) \mu i(\bar{s})$, we use the characterization of $\mu$. First note that as $(v(\bar{x}), i(\bar{x})) \in \operatorname{Idem}(\mathscr{E})$ we have that $\left(v^{*}(\bar{x}), j^{*}(\bar{x})\right) \in \operatorname{Idem}(\mathscr{E})$ where $j^{*}(\bar{x})=$ 
$v^{*}(\bar{x}) v(\bar{x}) i^{*}(\bar{x}) v(\bar{x}) v^{*}(\bar{x})$. Furthermore, it is easily seen that $j^{*}(\bar{s}) \in V(i(\bar{s}))$. Now let $y$ be an element of $X$ not appearing in $\bar{x}$ and let $e \in E$. (In any bi-identity involving $y$, both $y$ and $y^{*}$ will be replaced by $e$.) Clearly $\left(v(\bar{x}) y y^{*} v^{*}(\bar{x}), i(\bar{x}) y y^{*} j^{*}(\bar{x})\right) \epsilon$ $\operatorname{Idem}(\mathscr{E})$ and so, by the above result, it follows that $v(\bar{s}) e v^{*}(\bar{s}) \mathscr{H} i(\bar{s}) e j^{*}(\bar{s})$. Since both $v(\bar{s}) e v^{*}(\bar{s})$ and $i(\bar{s}) e j^{*}(\bar{s})$ are idempotents, we have in fact that $v(\bar{s}) e v^{*}(\bar{s})=$ $i(\bar{s}) e j^{*}(\bar{s})$. Similarly $v^{*}(\bar{s}) e v(\bar{s})=j^{*}(\bar{s}) e i(\bar{s})$. By the characterization of $\mu$, we have that $v(\bar{s}) \mu i(\bar{s})$ as required.

We now determine bi-identities for the Mal'cev product $\mathscr{U} \circ \mathscr{E}$, where $\mathscr{U} \in \mathscr{L}(\mathscr{G})$ and $\mathscr{E} \in \mathscr{L}_{e}(\mathscr{O})$. To do this we will need the concept of a verbal subsemigroup.

Let $\mathscr{E} \in \mathscr{L}_{e}(\mathscr{O}), V=\operatorname{Idem}(\mathscr{E})$ and $S \in \mathscr{O}$. The verbal subsemigroup of $S$ is defined by

$$
V(S)=\left\{v(\bar{x}) \alpha \mid \alpha \in \operatorname{Hom}\left(F^{*}(X), S\right) \text { and }(v(\bar{x}), i(\bar{x})) \in V\right\} .
$$

That $V(S)$ is in fact a subsemigroup of $S$ is shown in the following lemma.

LEMMA 6.6. Let $\mathscr{E} \in \mathscr{L}_{e}(\mathscr{O}), V=\operatorname{Idem}(\mathscr{E})$ and $S \in \mathscr{O}$. Then $V(S)$ is a full self-conjugate regular subsemigroup of $S$.

Proof. Let $a, b \in V(S)$. Then there are homomorphisms $\alpha, \beta \in \operatorname{Hom}\left(F^{*}(X), S\right)$ and $(v(\bar{x}), i(\bar{x})),(w(\bar{y}), j(\bar{y})) \in V$ where $\bar{x}=x_{1}, x_{1}^{*}, \ldots, x_{n}, x_{n}^{*}$ and $\bar{y}=x_{n+1}$, $x_{n+1}^{*}, \ldots, x_{n+m}, x_{n+m}^{*}$ such that $a=v(\bar{x}) \alpha$ and $b=w(\bar{y}) \beta$. It is clear that $(v(\bar{x}) w(\bar{y}), i(\bar{x}) j(\bar{y})) \in V$. Define a matched mapping $\chi: X \cup X^{*} \rightarrow S$ by

$$
\chi: x_{i}, x_{i}^{*} \mapsto \begin{cases}x_{i} \alpha, x_{i}^{*} \alpha & i=1, \ldots, n \\ x_{i} \beta, x_{i}^{*} \beta & i=n+1, \ldots, n+m \\ x_{1} \alpha, x_{1}^{*} \alpha & \text { otherwise }\end{cases}
$$

and let $\Phi: F^{*}(X) \rightarrow S$ be the unique homomorphism extending $\chi$. Then $(v(\bar{x}) w(\bar{y})) \Phi$ $=(v(\bar{x}) \Phi)(w(\bar{y}) \Phi)=(v(\bar{x}) \alpha)(w(\bar{y}) \beta)=a b$ is an element of $V(S)$. Thus $V(S)$ is closed under multiplication and hence is a subsemigroup of $S$.

As $(v(\bar{x}), i(\bar{x})) \in V$ it is easily seen that $\left(v^{*}(\bar{x}), v^{*}(\bar{x}) v(\bar{x}) i^{*}(\bar{x}) v(\bar{x}) v^{*}(\bar{x})\right) \in$ $V$. Hence $v^{*}(\bar{x}) \alpha \in V(S)$ is an inverse of $a=v(\bar{x}) \alpha$. Thus $V(S)$ is a regular subsemigroup of $S$.

To show that $V(S)$ is self-conjugate let $s \in S, s^{\prime} \in V(s)$. As $(v(\bar{x}), i(\bar{x})) \in V$ it is clear that $\left(x_{n+1}^{*} v(\bar{x}) x_{n+1}, x_{n+1}^{*} i(\bar{x}) x_{n+1}\right) \in V$. Define a matched mapping $\chi$ : $X \cup X^{*} \rightarrow S$ by

$$
\chi: x_{i}, x_{i}^{*} \mapsto \begin{cases}x_{i} \alpha, x_{i}^{*} \alpha & i=1, \ldots, n \\ s, s^{\prime} & i=n+1 \\ x_{1} \alpha, x_{1}^{*} \alpha & \text { otherwise }\end{cases}
$$


and let $\Phi: F^{*}(X) \rightarrow S$ be the unique homomorphism extending $\chi$. Then $\left(x_{n+1}^{*} v(\bar{x})\right.$ $\left.x_{n+1}\right) \Phi=\left(x_{n+1}^{*} \Phi\right)(v(\bar{x}) \Phi)\left(x_{n+1} \Phi\right)=s^{\prime} a s$ is an element of $V(S)$. It follows that $s^{\prime} V(S) s \subseteq V(S)$ and so $V(S)$ is self-conjugate.

For any $e \in E$, let $\alpha: F^{*}(X) \rightarrow S$ be defined by $\alpha: v(\bar{x}) \mapsto e\left(v(\bar{x}) \in F^{*}(X)\right)$. Then, as $\left(x x^{*}, x x^{*}\right) \in V$, we see that $e=\left(x x^{*}\right) \alpha \in V(S)$ and $V(S)$ is full.

THEOREM 6.7. Let $\mathscr{U} \in \mathscr{L}(\mathscr{G})$ and $\mathscr{E} \in \mathscr{L}_{e}(\mathscr{O})$. Then $S \in \mathscr{U} \circ \mathscr{E}$ if and only if $S$ satisfies all bi-identities of the following forms:

(a) $v(\bar{x}) v^{*}(\bar{x}) i(\bar{x})=i(\bar{x})=i(\bar{x}) v^{*}(\bar{x}) v(\bar{x})$, where $(v(\bar{x}), i(\bar{x})) \in \operatorname{Idem}(\mathscr{E})$;

(b) $u\left(v_{1}\left(\bar{x}_{1}\right), v_{1}^{*}\left(\bar{x}_{1}\right), \ldots, v_{n}\left(\bar{x}_{n}\right), v_{n}^{*}\left(\bar{x}_{n}\right)\right)=u\left(i_{1}\left(\bar{x}_{1}\right), j_{1}^{*}\left(\bar{x}_{1}\right), \ldots, i_{n}\left(\bar{x}_{n}\right), j_{n}^{*}\left(\bar{x}_{n}\right)\right)$, where $u\left(x_{1}, x_{1}^{-1}, \ldots, x_{n}, x_{n}^{-1}\right) \in \operatorname{Id}(\mathscr{U}),\left(v_{k}\left(\bar{x}_{k}\right), i_{k}\left(\bar{x}_{k}\right)\right) \in \operatorname{Idem}(\mathscr{E})$ and $j_{k}^{*}\left(\bar{x}_{k}\right)=$ $v_{k}^{*}\left(\bar{x}_{k}\right) v_{k}\left(\bar{x}_{k}\right) i_{k}^{*}\left(\bar{x}_{k}\right) v_{k}\left(\bar{x}_{k}\right) v_{k}^{*}\left(\bar{x}_{k}\right)$.

PROOF. Let $S \in \mathscr{U} \circ \mathscr{E}$. Then $S \in \mathscr{G} \circ \mathscr{E}$ and so $S$ satisfies all bi-identities of the forms given in (a) by Theorem 6.5 .

Let $\theta$ be a congruence on $S$ such that $S / \theta \in \mathscr{E}$ and $e \theta \in \mathscr{U}$ for each $e \in$ $E$. Let $u\left(x_{1}, x_{1}^{-1}, \ldots, x_{n}, x_{n}^{-1}\right) \in \operatorname{Id}(\mathscr{U}),\left(v_{k}\left(\bar{x}_{k}\right), i_{k}\left(\bar{x}_{k}\right)\right) \in \operatorname{Idem}(\mathscr{E})$ and $j_{k}^{*}\left(\bar{x}_{k}\right)=$ $v_{k}^{*}\left(\bar{x}_{k}\right) v_{k}\left(\bar{x}_{k}\right) i_{k}^{*}\left(\bar{x}_{k}\right) v_{k}\left(\bar{x}_{k}\right) v_{k}^{*}\left(\bar{x}_{k}\right)$. Note that as $\left(v_{k}\left(\bar{x}_{k}\right), i_{k}\left(\bar{x}_{k}\right)\right) \in \operatorname{Idem}(\mathscr{E})$, we have that $\left(v_{k}^{*}\left(\bar{x}_{k}\right), j_{k}^{*}\left(\bar{x}_{k}\right)\right) \in \operatorname{Idem}(\mathscr{E})$. Let $\bar{s}_{k}=s_{k}^{1},\left(s_{k}^{1}\right)^{\prime}, \ldots, s_{k}^{m(k)},\left(s_{k}^{m(k)}\right)^{\prime}$ be a string of elements of $S$, where $\left(s_{k}^{i}\right)^{\prime} \in V\left(s_{k}^{i}\right)$. As $\left(v_{k}\left(\bar{x}_{k}\right), i_{k}\left(\bar{x}_{k}\right)\right),\left(v_{k}^{*}\left(\bar{x}_{k}\right), j_{k}^{*}\left(\bar{x}_{k}\right)\right) \in \operatorname{Idem}(\mathscr{E})$ and $S / \theta \in \mathscr{E}$, we have that $v_{k}\left(\bar{s}_{k}\right) \theta=i_{k}\left(\bar{s}_{k}\right) \theta$ and $v_{k}^{*}\left(\bar{s}_{k}\right) \theta=j_{k}^{*}\left(\bar{s}_{k}\right) \theta$. Hence

$$
\begin{aligned}
u\left(v_{1}\left(\bar{s}_{1}\right), v_{1}^{*}\left(\bar{s}_{1}\right), \ldots, v_{n}\left(\bar{s}_{n}\right), v_{n}^{*}\left(\bar{s}_{n}\right)\right) \theta & =u\left(v_{1}\left(\bar{s}_{1}\right) \theta, v_{1}^{*}\left(\bar{s}_{1}\right) \theta, \ldots, v_{n}\left(\bar{s}_{n}\right) \theta, v_{n}^{*}\left(\bar{s}_{n}\right) \theta\right) \\
& =u\left(i_{1}\left(\bar{s}_{1}\right) \theta, j_{1}^{*}\left(\bar{s}_{1}\right) \theta, \ldots, i_{n}\left(\bar{s}_{n}\right) \theta, j_{n}^{*}\left(\bar{s}_{n}\right) \theta\right) \\
& =u\left(i_{1}\left(\bar{s}_{1}\right), j_{1}^{*}\left(\bar{s}_{1}\right), \ldots, i_{n}\left(\bar{s}_{n}\right), j_{n}^{*}\left(\bar{s}_{n}\right)\right) \theta
\end{aligned}
$$

Let $e=u\left(i_{1}\left(\bar{s}_{1}\right), j_{1}^{*}\left(\bar{s}_{1}\right), \ldots, i_{n}\left(\bar{s}_{n}\right), j_{n}^{*}\left(\bar{s}_{n}\right)\right)$. Then, as $i_{k}\left(\bar{s}_{k}\right), j_{k}^{*}\left(\bar{s}_{k}\right)$ are idempotents of $S, e$ is an idempotent of $S$.

As $\operatorname{Ker} \theta$ is a band of groups, $\operatorname{Ker} \theta / \gamma$ is a semilattice of groups. By [8, Theorem IV.2.1] the idempotents of a semilattice of groups are central. Therefore, as $v_{k}\left(\bar{s}_{k}\right)$, $v_{k}^{*}\left(\bar{s}_{k}\right) \in \operatorname{Ker} \theta$ and $u\left(v_{1}\left(\bar{s}_{1}\right), v_{1}^{*}\left(\bar{s}_{1}\right), \ldots, v_{n}\left(\bar{s}_{n}\right), v_{n}^{*}\left(\bar{s}_{n}\right)\right) \in e \theta$ which is a group with identity $e$ we have that

$$
\begin{aligned}
u\left(v_{1}\left(\bar{s}_{1}\right), v_{1}^{*}\left(\bar{s}_{1}\right), \ldots, v_{n}\left(\bar{s}_{n}\right), v_{n}^{*}\left(\bar{s}_{n}\right)\right)= & e \cdot u\left(v_{1}\left(\bar{s}_{1}\right), v_{1}^{*}\left(\bar{s}_{1}\right), \ldots, v_{n}\left(\bar{s}_{n}\right), v_{n}^{*}\left(\bar{s}_{n}\right)\right) \\
& \gamma u\left(e v_{1}\left(\bar{s}_{1}\right) e, e v_{1}^{*}\left(\bar{s}_{1}\right) e, \ldots, e v_{n}\left(\bar{s}_{n}\right) e, e v_{n}^{*}\left(\bar{s}_{n}\right) e\right)
\end{aligned}
$$

We show that $e v_{k}\left(\bar{s}_{k}\right) e, e v_{k}^{*}\left(\bar{s}_{k}\right) e \in e \theta$.

By the first paragraph, $S$ satisfies all bi-identities of the forms given in (a). Therefore,

$$
j_{k}^{*}\left(\bar{s}_{k}\right)=v_{k}^{*}\left(\bar{s}_{k}\right) v_{k}\left(\bar{s}_{k}\right) i_{k}^{*}\left(\bar{s}_{k}\right) v_{k}\left(\bar{s}_{k}\right) v_{k}^{*}\left(\bar{s}_{k}\right)
$$




$$
\begin{aligned}
& \gamma v_{k}^{*}\left(\bar{s}_{k}\right) v_{k}\left(\bar{s}_{k}\right) i_{k}\left(\bar{s}_{k}\right) v_{k}\left(\bar{s}_{k}\right) v_{k}^{*}\left(\bar{s}_{k}\right) \\
& \gamma v_{k}\left(\bar{s}_{k}\right) v_{k}^{*}\left(\bar{s}_{k}\right) i_{k}\left(\bar{s}_{k}\right) v_{k}^{*}\left(\bar{s}_{k}\right) v_{k}\left(\bar{s}_{k}\right)=i_{k}\left(\bar{s}_{k}\right) .
\end{aligned}
$$

Furthermore, it is easily seen that $e \gamma i_{1}\left(\bar{s}_{1}\right) \cdots i_{n}\left(\bar{s}_{n}\right)$. It follows that $e i_{k}\left(\vec{s}_{k}\right) e \gamma e$ and $e j_{k}^{*}\left(\bar{s}_{k}\right) e \gamma e$. By the characterization of $\gamma, V\left(e i_{k}\left(\bar{s}_{k}\right) e\right)=V(e)$, and so $e i_{k}\left(\bar{s}_{k}\right) e=$ $e \cdot e i_{k}\left(\bar{s}_{k}\right) e \cdot e=e$, and similarly $e j_{k}^{*}\left(\bar{s}_{k}\right) e=e$. Therefore $e v_{k}\left(\bar{s}_{k}\right) e \theta e i_{k}\left(\bar{s}_{k}\right) e=e$ and $e v_{k}^{*}\left(\bar{s}_{k}\right) e \theta e j_{k}^{*}\left(\bar{s}_{k}\right) e=e$.

We now have that $e v_{k}\left(\bar{s}_{k}\right) e \in e \theta$ and $e v_{k}^{*}\left(\bar{s}_{k}\right) e$ is the inverse of $e v_{k}\left(\bar{s}_{k}\right) e$ in the group $e \theta$. Since $e \theta \in \mathscr{U}$ and $u\left(x_{1}, x_{1}^{-1}, \ldots, x_{n}, x_{n}^{-1}\right) \in \operatorname{Id}(\mathscr{U})$, we have that $u\left(e v_{1}\left(\bar{s}_{1}\right) e, e v_{1}^{*}\left(\bar{s}_{1}\right) e, \ldots, e v_{n}\left(\bar{s}_{n}\right) e, e v_{n}^{*}\left(\bar{s}_{n}\right) e\right)=e$. Therefore $u\left(v_{1}\left(\bar{s}_{1}\right), v_{1}^{*}\left(\bar{s}_{1}\right), \ldots\right.$, $\left.v_{n}\left(\bar{s}_{n}\right), v_{n}^{*}\left(\bar{s}_{n}\right)\right) \gamma e$ and hence $u\left(v_{1}\left(\bar{s}_{1}\right), v_{1}^{*}\left(\bar{s}_{1}\right), \ldots, v_{n}\left(\bar{s}_{n}\right), v_{n}^{*}\left(\bar{s}_{n}\right)\right)$ is an idempotent of $S$, since $\gamma$ is idempotent pure. Finally, as $u\left(v_{1}\left(\bar{s}_{1}\right), v_{1}^{*}\left(\bar{s}_{1}\right), \ldots, v_{n}\left(\bar{s}_{n}\right), v_{n}^{*}\left(\bar{s}_{n}\right)\right) \in e \theta$, which is a group with identity $e$, we have that

$$
u\left(v_{1}\left(\bar{s}_{1}\right), v_{1}^{*}\left(\bar{s}_{1}\right), \ldots, v_{n}\left(\bar{s}_{n}\right), v_{n}^{*}\left(\bar{s}_{n}\right)\right)=e=u\left(i_{1}\left(\bar{s}_{1}\right), j_{1}^{*}\left(\bar{s}_{1}\right), \ldots, i_{n}\left(\bar{s}_{n}\right), j_{n}^{*}\left(\bar{s}_{n}\right)\right) .
$$

Thus $S$ satisfies all bi-identities of the forms given in (b).

For the converse, let $S$ be an orthodox semigroup satisfying all bi-identities of the forms given in the statement of the theorem and let $V=\operatorname{Idem}(\mathscr{E})$. Let $a \in V(S)$. Then there is $(v(\bar{x}), i(\bar{x})) \in V$ and a string of elements $\bar{s}=s_{1}, s_{1}^{\prime}, \ldots, s_{n}, s_{n}^{\prime}$ of $S$, where $s_{i}^{\prime} \in V\left(s_{i}\right)$, such that $a=v(\bar{s})$. Since $S$ satisfies the bi-identities of the forms given in (a), $S / \mu \in \mathscr{E}$ by Theorem 6.5 and therefore $S / \mu$ satisfies the bi-identity $v(\bar{x})=i(\bar{x})$. Now $a \mu=v(\bar{s}) \mu=i(\bar{s}) \mu$, that is, $a$ is $\mu$-related to the idempotent $i(\bar{s})$. Hence $a \in \operatorname{Ker} \mu$ and so $V(S) \subseteq \operatorname{Ker} \mu$. By Lemma $6.6, V(S)$ is a full self-conjugate regular subsemigroup of $S$ and therefore $V(S)$ is the kernel of an i.s. congruence, $\theta$ say, on $S$, by Theorem 2.10. We show that $S / \theta \in \mathscr{E}$ and $e \theta \in \mathscr{U}$ for each $e \in E$.

To show that $S / \theta \in \mathscr{E}$ it suffices to show that $v(\bar{s}) \theta i(\bar{s})$, where $(v(\bar{x}), i(\bar{x})) \in$ $\operatorname{Idem}(\mathscr{E})$ and $\bar{s}=s_{1}, s_{1}^{\prime}, \ldots, s_{n}, s_{n}^{\prime}$ is a string of elements of $S$ with $s_{i}^{\prime} \in V\left(s_{i}\right)$. Since $S$ satisfies the bi-identities of the forms given in (a), we have that $v(\bar{s}) v^{*}(\bar{s}) i(\bar{s})=i(\bar{s})=$ $i(\bar{s}) v^{*}(\bar{s}) v(\bar{s})$, and also $i(\bar{s}) i^{*}(\bar{s}) v(\bar{s})=v(\bar{s})=v(\bar{s}) i^{*}(\bar{s}) i(\bar{s})$ (since $\left(v^{*}(\bar{x}) i(\bar{x}), v^{*}(\bar{x})\right.$ $v(\bar{x})) \in \operatorname{Idem}(\mathscr{E})$ as in the proof of Theorem 6.5). Now

$$
\begin{aligned}
v(\bar{s}) v^{*}(\bar{s}) & =i(\bar{s}) i^{*}(\bar{s}) v(\bar{s}) v^{*}(\bar{s}) \\
& =i(\bar{s}) v^{*}(\bar{s}) v(\bar{s}) i^{*}(\bar{s}) v(\bar{s}) v^{*}(\bar{s})=i(\bar{s}) j^{*}(\bar{s})
\end{aligned}
$$

and

$$
\begin{aligned}
v^{*}(\bar{s}) v(\bar{s}) & =v^{*}(\bar{s}) v(\bar{s}) i^{*}(\bar{s}) i(\tilde{s}) \\
& =v^{*}(\bar{s}) v(\bar{s}) i^{*}(\bar{s}) v(\bar{s}) v^{*}(\bar{s}) i(\bar{s})=j^{*}(\bar{s}) i(\bar{s}) .
\end{aligned}
$$

Furthermore it is easily seen that $v(\bar{s}) j^{*}(\bar{s}), v^{*}(\bar{s}) i(\bar{s}) \in V(S)$ and that $j^{*}(\bar{s}) \in$ $V(i(\bar{s}))$. Therefore, by the characterization of $\theta$ (Theorem 2.10) we have $v(\bar{s}) \theta i(\bar{s})$ as required. 
Finally let $e \in E, u\left(x_{1}, x_{1}^{-1}, \ldots, x_{n}, x_{n}^{-1}\right) \in \operatorname{Id}(\mathscr{U})$ and $g_{1}, \ldots, g_{n} \in e \theta$. Since $g_{k} \in \operatorname{Ker} \theta=V(S)$ each $g_{k}$ has the form $v_{k}\left(\bar{x}_{k}\right) \alpha_{k}$ where $\alpha_{k} \in \operatorname{Hom}\left(F^{*}(X), S\right)$ and $\left(v_{k}\left(\bar{x}_{k}\right), i_{k}\left(\bar{x}_{k}\right)\right) \in V$. Without loss of generality we can assume that the distinct strings $\bar{x}_{k}$ have no element in common. Define $\alpha \in \operatorname{Hom}\left(F^{*}(X), S\right)$ as an extension of the map $\chi$ determines as follows: for each $y$ in the $k$-th string $\bar{x}_{k}$ let $y \chi=y \alpha_{k}$ ( $\chi$ is well-defined since the distinct strings $\bar{x}_{k}$ have no element in common). Then $v_{k}\left(\bar{x}_{k}\right) \alpha=v_{k}\left(\bar{x}_{k}\right) \alpha_{k}=g_{k}$ and $v_{k}^{*}\left(\bar{x}_{k}\right) \alpha$ is an inverse $g_{k}^{\prime}$ say of $g_{k}$. Let $g_{k}^{-1}$ be the inverse of $g_{k}$ in the group $e \theta$. Then $g_{k}^{-1} \gamma=\left(g_{k} \gamma\right)^{-1}=g_{k}^{\prime} \gamma=v_{k}^{*}\left(\bar{x}_{k}\right) \alpha \gamma$. Hence

$$
\begin{aligned}
u\left(g_{1}, g_{1}^{-1}, \ldots, g_{n}, g_{n}^{-1}\right) \gamma & =u\left(g_{1} \gamma, g_{1}^{-1} \gamma, \ldots, g_{n} \gamma, g_{n}^{-1} \gamma\right) \\
& =u\left(v_{1}\left(\bar{x}_{1}\right) \alpha \gamma, v_{1}^{*}\left(\bar{x}_{1}\right) \alpha \gamma, \ldots, v_{n}\left(\bar{x}_{n}\right) \alpha \gamma, v_{n}^{*}\left(\bar{x}_{n}\right) \alpha \gamma\right) \\
& =u\left(v_{1}\left(\bar{x}_{1}\right), v_{1}^{*}\left(\bar{x}_{1}\right), \ldots, v_{n}\left(\bar{x}_{n}\right), v_{n}^{*}\left(\bar{x}_{n}\right)\right) \alpha \gamma \\
& =u\left(i_{1}\left(\bar{x}_{1}\right), j_{1}^{*}\left(\bar{x}_{1}\right), \ldots, i_{n}\left(\bar{x}_{n}\right), j_{n}^{*}\left(\bar{x}_{n}\right)\right) \alpha \gamma
\end{aligned}
$$

since by assumption $S$ satisfies bi-identities of the form given in (b). Thus $u\left(g_{1}, g_{1}^{-1}, \ldots\right.$, $\left.g_{n}, g_{n}^{-1}\right) \gamma$ is an idempotent of $S / \gamma$ and hence $u\left(g_{1}, g_{1}^{-1}, \ldots, g_{n}, g_{n}^{-1}\right)$ is an idempotent of $S$ and in fact of $e \theta$. Since $e \theta$ is a group with identity $e$ this yields that $u\left(g_{1}, g_{1}^{-1}, \ldots, g_{n}, g_{n}^{-1}\right)=e$. We have shown that $e \theta$ satsifies all the identities of $\operatorname{Id}(\mathscr{U})$, thus $e \theta \in \mathscr{U}$ for all $e \in E$. Hence $\theta$ is a congruence on $S$ such that $S / \theta \in \mathscr{E}$ and $e \theta \in \mathscr{U}$ for all $e \in E$, and so $S \in \mathscr{U} \circ \mathscr{E}$.

\section{Acknowledgements}

I would like to thank T. E. Hall for his suggestion of this topic for research and for his useful suggestions about this paper, P. R. Jones for pointing out that cryptic evarieties are completely semisimple and the referee for their comments which greatly improved this paper.

\section{References}

[1] J. L. Bales, 'On product varieties of inverse semigroups', J. Austral. Math. Soc. (Series A) 28 (1979), 107-119.

[2] R. Feigenbaum, Kernels of regular semigroup homomorphisms (Ph.D. Thesis, University of South Carolina, 1975).

[3] — , 'Kernels of orthodox semigroup homomorphisms', J. Austral. Math. Soc. (Series A) 22 (1976), 234-245.

[4] __-, 'Regular semigroup congruences', Semigroup Forum 17 (1979), 373-377.

[5] T. E. Hall, 'Congruences and Greens relations on regular semigroups', Glasgow Math.J. 13 (1972), 167-175. 
[6] - 'On regular semigroups', J. Algebra 24 (1973), 1-24.

[7] — 'Identities for existence varieties of regular semigroups', Bull. Austral. Math. Soc. 40 (1989), 59-77.

[8] J. M. Howie, An introduction to semigroup theory (Academic Press, London, 1976).

[9] J. M. Howie and G. Lallement, 'Certain fundamental congruences on a regular semigroup', Proc. Glasgow Math. Assoc. 7 (1976), 145-159.

[10] J. Kad'ourek and M. B. Szendrai, 'A new approach in the theory of orthodox semigroups', Semigroup Forum 40 (1990), 257-296.

[11] E. I. Kleiman, 'On the lattice of varieties of inverse semigroups', Izv. Vyssh. Ucebn. Zaved. Mat. 7 (1976), 106-109.

[12] D. R. La Torre, 'Group congruences on regular semigroups', Semigroup Forum 24 (1982), 327 340.

[13] J. Meakin, 'Congruences on orthodox semigrous', J. Austral. Math. Soc. 12 (1971), 323-341.

[14] — - 'Congruences on orthodox semigrous II', J. Austral. Math. Soc. 13 (1972), 259-266.

[15] F. Pastijn and M. Petrich, 'Congruences on regular semigroups', Trans. Amer. Math. Soc. 295 (1986), 607-633.

[16] F. Pastijn and P. G. Trotter, 'Lattices of completely regular semigroup varieties', Pacific J. Math. 119 (1985), 191-214.

[17] M. Petrich, Introduction to semigroups (Merrill, Columbus, 1973).

[18] - Inverse semigroups (Wiley, New York, 1984).

[19] N. R. Reilly, 'Modular sublattices of the lattice of varieties of inverse semigroups', Pacific J. Math. $89(1980), 405-417$.

[20] — ' 'Varieties of completely semisimple inverse semigroups', J. Algebra 65 (1980), 427-444.

[21] _ 'Minimal non-cryptic varieties of inverse semigroups', Quart. J. Math. Oxford Ser. (2) 36 (1985), 467-487.

[22] N. R. Reilly and H. E. Scheiblich, 'Congruences on regular semigroups', Pacific J. Math. 23 (1967), 349-360.

[23] T. Saito, 'Ordered regular proper semigroups', J. Algebra 8 (1968), 450-477.

[24] H. E. Scheiblich, 'Certain congruence and quotient lattices related to completely 0-simple and primitive regular semigroups', Glasgow Math. J. 10 (1969), 21-24.

[25] M. B. Szendrai, 'On a pull-back diagram for orthodox semigroups', Semigroup Forum 20 (1980), 1-10 Corrigendum: 25 (1982), 311-324.

[26] _ ' 'Free *-orthodox semigroups', Simon Stevin 59 (1985), 175-201.

\section{Department of Mathematics}

Monash University

Clayton, Vic. 3168

Australia 